\title{
Differentiation of hypothalamic-like neurons from human pluripotent stem cells
}

\author{
Liheng Wang, ${ }^{1,2}$ Kana Meece, ${ }^{3}$ Damian J. Williams, ${ }^{4}$ Kinyui Alice Lo, ${ }^{5}$ Matthew Zimmer, ${ }^{6}$ Garrett Heinrich, ${ }^{3}$ Jayne Martin Carli, ${ }^{3}$ \\ Charles A. Leduc, ${ }^{1,3}$ Lei Sun, ${ }^{5,7}$ Lori M. Zeltser, ${ }^{1,2}$ Matthew Freeby, ${ }^{3}$ Robin Goland, ${ }^{3}$ Stephen H. Tsang, ${ }^{2,8}$ Sharon L. Wardlaw, ${ }^{3}$ \\ Dieter Egli, ${ }^{1,3,6}$ and Rudolph L. Leibel ${ }^{1,2,3}$ \\ 'Division of Molecular Cenetics, Department of Pediatrics and Naomi Berrie Diabetes Center, Columbia University College of Physicians and Surgeons, New York, New York, USA. ${ }^{2}$ Institute of Human Nutrition, \\ Columbia University, New York, New York, USA. Department of Medicine and Naomi Berrie Diabetes Center, Columbia University College of Physicians and Surgeons, New York, New York, USA. \\ ${ }^{4}$ Department of Pathology and Cell Biology, Columbia University, New York, New York, USA. ${ }^{5}$ Institute of Molecular and Cell Biology, Proteos, Singapore. ${ }^{6}$ New York Stem Cell Foundation Research Institute, \\ New York, New York, USA. ${ }^{7}$ Cardiovascular and Metabolic Disorders Program, Duke-NUS, Singapore. ${ }^{8}$ Barbara and Donald Jonas Laboratory of Stem Cells and Regenerative Medicine, \\ and Bernard and Shirlee Brown Claucoma Laboratory, Department of Ophthalmology, Columbia University, New York, New York, USA.
}

\begin{abstract}
The hypothalamus is the central regulator of systemic energy homeostasis, and its dysfunction can result in extreme body weight alterations. Insights into the complex cellular physiology of this region are critical to the understanding of obesity pathogenesis; however, human hypothalamic cells are largely inaccessible for direct study. Here, we developed a protocol for efficient generation of hypothalamic neurons from human embryonic stem cells (ESCs) and induced pluripotent stem cells (iPSCs) obtained from patients with monogenetic forms of obesity. Combined early activation of sonic hedgehog signaling followed by timed NOTCH inhibition in human ESCs/iPSCs resulted in efficient conversion into hypothalamic NKX2.1+ precursors. Application of a NOTCH inhibitor and brain-derived neurotrophic factor (BDNF) further directed the cells into arcuate nucleus hypothalamic-like neurons that express hypothalamic neuron markers proopiomelanocortin (POMC), neuropeptide Y (NPY), agouti-related peptide (AGRP), somatostatin, and dopamine. These hypothalamic-like neurons accounted for over $\mathbf{9 0 \%}$ of differentiated cells and exhibited transcriptional profiles defined by a hypothalamic-specific gene expression signature that lacked pituitary markers. Importantly, these cells displayed hypothalamic neuron characteristics, including production and secretion of neuropeptides and increased p-AKT and p-STAT3 in response to insulin and leptin. Our results suggest that these hypothalamic-like neurons have potential for further investigation of the neurophysiology of body weight regulation and evaluation of therapeutic targets for obesity.
\end{abstract}

\section{Introduction}

The mediobasal hypothalamus is a functional integrator of homeostatic processes, including food intake, energy expenditure, neuroendocrine regulation, body temperature, and circadian rhythms (1). Constituent cell bodies with distinct physiological functions include the arcuate ventromedial (VMH), dorsal medial (DMH), and paraventricular (PVH) nuclei $(2,3)$. Arcuate nucleus (ARC) neurons, such as those expressing proopiomelanocortin (POMC) and neuropeptide Y (NPY)/agouti-related peptide (AGRP), can sense peripheral hormones - insulin, leptin, ghrelin, PYY - and secrete neuropeptides $\alpha$ melanocyte-stimulating hormone $(\alpha \mathrm{MSH})$ and NPY/AGRP to engage receptors on so-called "second order" DMH, $\mathrm{PVH}$, and other neurons to regulate aspects of energy homeostasis through melanocortin 4 receptor (MC4R), neuropeptide Y receptor type 1 (NPY1R), and other receptors (3). Hypomorphic mutations of genes involved in hypothalamic leptin-melanocortin signaling, such as leptin, leptin receptor, POMC, and MC4R, result in monogenic severe obesity in humans and rodents, confirming the biological importance of these pathways(4-8). Though various neuro-

Conflict of interest: The authors have declared that no conflict of interest exists. Submitted: October 2, 2014; Accepted: November 20, 2014.

Reference information: / Clin Invest. 2015;125(2):796-808. doi:10.1172/JCI79220. nal cell types have been generated by directed differentiation from human pluripotent stem cells and applied for the study of neurodegenerative diseases, including Alzheimer's disease, Parkinson's disease, and ALS (9-11), there is currently no published protocol for the differentiation of human hypothalamic neurons. For the analysis of obesity-related pathophysiology, access to hypothalamic cell types would be extremely useful.

Establishing directed differentiation protocols relies upon an understanding of the details of cellular ontogenesis. The hypothalamus is a complex organ subserving roles in energy homeostasis, endocrine physiology, temperature regulation, arousal, circadian rhythms, and other functions that are mediated by specific hypothalamic cell types (12). A growing number of transcription factors (TFs) have been implicated in the differentiation and specification of hypothalamic neuronal subtypes (Supplemental Figure 1A; supplemental material available online with this article; doi:10.1172/ JCI79220DS1). In the E10.5 mouse brain, Nkx2.1 is expressed in both the ventral diencephalon and telencephalon, while FoxG1 is expressed in telencephalon progenitors, but not in hypothalamic progenitors, suggesting that hypothalamic neurons likely develop from Nkx2.1 $1^{+}$FoxG1- precursors (13). RAX, VAX, and SIX3 are specifically expressed in rostral hypothalamic neuroepithelia (14, 15). Moreover, Achaete-scute-like 1 (ASCL1, also called MASH1), 
Nescient helix loop helix 2 (NHLH2), and Orthopedia (OTP) are involved in specifying ARC neurons, including POMC, NPY, AGRP, GHRH, and dopaminergic (DA) neurons (16-18). SIM1, Aryl hydrocarbon receptor nuclear translocator 2 (ARNT2), OTP, and POU3F2 are required for the expression of oxytocin, vasopressin (AVP), TRH, and CRH in PVH neurons $(19,20)$; steroidogenic factor-1 (SF1) and ASCL1 are important for the generation of VMH neurons (21). However, signals or pathways that participate in regulating the expression of these TFs are not well understood. We do know, however, that sonic hedgehog (SHH), a secreted ventralization morphogen, is essential for the induction and patterning of the hypothalamus $(2,22)$.

These fundamental aspects of hypothalamic development guided our development of a differentiation protocol for the efficient generation of hypothalamic ARC-like neurons from human embryonic stem cells (hESCs)/induced pluripotent stem cells (iPSCs). With early activation of $\mathrm{SHH}$ signaling plus SMAD dual inhibition followed by inhibition of Notch signaling, we were able to convert human ESCs into ventral diencephalon NKX2.1 ${ }^{+} \mathrm{FOXG1} 1^{-}$progenitors. We then further differentiated these progenitors into hypothalamic ARC-like neurons by inhibition of Notch signaling and demonstrated hypothalamic-specific gene signatures by RNA-seq and quantitative PCR (qPCR). We found that these neurons expressed and accurately processed hypothalamic peptides, including POMC, $\alpha \mathrm{MSH}, \beta$-endorphin (BEP), and AGRP, and we demonstrated that hESC-derived neurons could sense and respond to metabolic signals, including insulin and leptin. Importantly, this protocol also works efficiently and consistently for generating hypothalamic neurons from human iPSC lines derived from both healthy controls and subjects with obesityrelated mutations such as Bardet-Biedl syndrome (BBS). Taken together, these findings suggest that we have generated neurons that closely resemble those of the ARC hypothalamus, providing a new resource for the study of the neuromolecular mechanisms for regulation of human body weight.

\section{Results}

Generation of hypothalamic NKX2.1 progenitors from hESCs. The hypothalamus develops from the ventral diencephalon, where NKX2.1 is highly expressed. High-dose SHH(C24II) treatment efficiently induces the expression of NKX2.1 in human ESCs (23, 24). The timing of SHH exposure influ-ences the development of the ventral neuron population in the mouse hypothalamus: early Shh-expressing progenitors contribute to neurons and astrocytes of the tuberal region, while late Shh-expressing progenitors almost exclusively generate astrocytes in mouse hypothalamus development (2). Therefore, we asked whether early exposure to high $\mathrm{SHH}$ could induce ventral diencephalon NKX2.1+FOXG1- progenitors from human ESCs.

Using an NKX2.1-GFP/W-hESC reporter line (20) (hereafter referred to as hESC), we varied the timing of $\mathrm{SHH}$ exposure to optimize conditions for NKX2.1-GFP expression (Supplemental Figure 1B). We adapted an embryoid body (EB) culture system for hESC differentiation, as was used for rostral hypothalamic neuron differentiation in mouse ESCs (24). We also used SMAD inhibitors - LDN193189 $(1 \mu \mathrm{M})$ and SB431542 (referred to hereafter as LSB) $(10 \mu \mathrm{M})$ - from days 1 to 8 to drive ESCs toward a neuronal lineage
(25). GFP expression was measured with fluorescence imaging or FACS. We found that activation of SHH signaling from days 3 to 13 was optimal with regard to NKX2.1-GFP expression in day-26 EBs (Supplemental Figure 1C). The addition of purmorphamine (PM) $(2 \mu \mathrm{M})$, a SHH agonist, with SHH (100 ng/ml) further promoted NKX2.1-GFP expression in day-26 EB (70.1\% GFP ${ }^{+}$cells) (Supplemental Figure 1D). Subsequently, we found that a monolayer feeder-free culture system significantly shortened the time required for generation of NKX2.1 progenitors (11-12 days versus 26 days) (Supplemental Figure 1E). This culture system was used for all subsequent studies.

Notch signaling controls progenitor maintenance and differentiation of hypothalamic neurons $(26,27)$. Loss of Notch signaling in Nkx2.1 progenitors promotes the differentiation of hypothalamic ARC neurons, and transient inhibition of Notch signaling synchronizes the differentiation of neuron progenitors $(26,28)$. Therefore, we asked whether inhibition of Notch signaling would promote the generation of hypothalamic progenitors. Three days of exposure to the Notch signaling inhibitor $\mathrm{N}$-[(3,5-difluorophenyl) acetyl]-L-alanyl-2-phenyl]glycine-1,1-dimethylethyl ester (DAPT) $(10 \mu \mathrm{M})$ (from day 8 to day 11), after withdrawal of SHH and LSB (Supplemental Figure 1F), increased the expression of many TFs that are required for hypothalamic neuron specification (ASCL1, NHLH2, SIM1, OTP) and the neuron progenitor marker NESTIN, but not the telencephalon markers PAX6 and FOXG1 (Supplemental Figure 1F). High levels of NKX2.1 expression persisted during this 3-day period. Continued SHH+LSB treatment did not induce any of these TF markers (Supplemental Figure 1F). Therefore, inhibition of Notch signaling after activation of SHH signaling, combined with dual SMAD inhibition, triggers the production of hypothalamic NKX2.1 progenitors.

With this protocol (Figure 1A), by immunohistochemistry, more than $90 \%$ of day-12-differentiated cells were NKX2.1 $1^{+}$, and less than $1 \%$ were $\mathrm{FOXG}^{+}$(Figure $1 \mathrm{~B}$ ), suggesting the production of ventral diencephalon-specific but not telencephalon progenitors. By qPCR of 12-day cells, we detected the upregulation of TFs that were expressed in hypothalamic progenitors: NKX2.1, MASH1, NHLH2, OTP, SIM1, SF1, and the neural progenitor marker NESTIN (Figure 1C). PAX6, an early forebrain TF gene, was reduced in day- 12 versus day- 8 cells. By immunostaining, we also found that PAX6 and FOXG1 expression ceased (Supplemental Figure 2), while MASH1 continued to be expressed in day-12 neural precursors (Figure 1D). Most day-12 cells also expressed SOX1 and NESTIN (Supplemental Figure 2), both of which are markers of neuronal progenitors. Such dynamic changes of these TFs were further confirmed by Western blot analysis (Figure 1E). Both MASH1 and NKX2.1 protein levels showed time-dependent increases from hESC to day-12 progenitors, while PAX6 was only transiently expressed in day- 8 cells (Figure 1E). Therefore, early activation of SHH signaling with LSB treatment and subsequent inhibition of Notch signaling provides a strategy for effectively generating hypothalamic NKX2.1 $1^{+}$neuronal progenitors.

Neurons derived from $N k x 2.1^{+}$progenitors recapitulate hypothalamic ARC identities. To advance the differentiation of day- 12 NKX2.1 $1^{+}$progenitors, we dissociated and replated these cells on poly-L-ornithine/laminin-coated plates. To induce hypothalamic ARC neuron differentiation, Notch signaling was inhibited using 
A

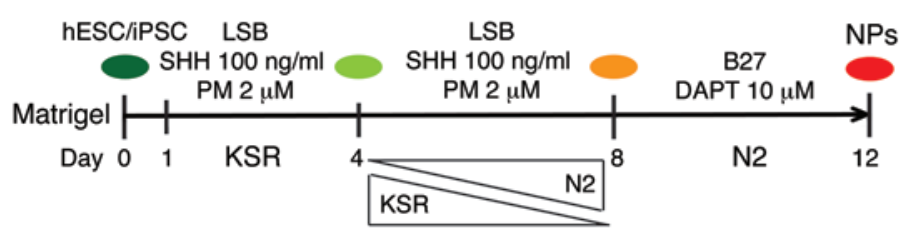

B
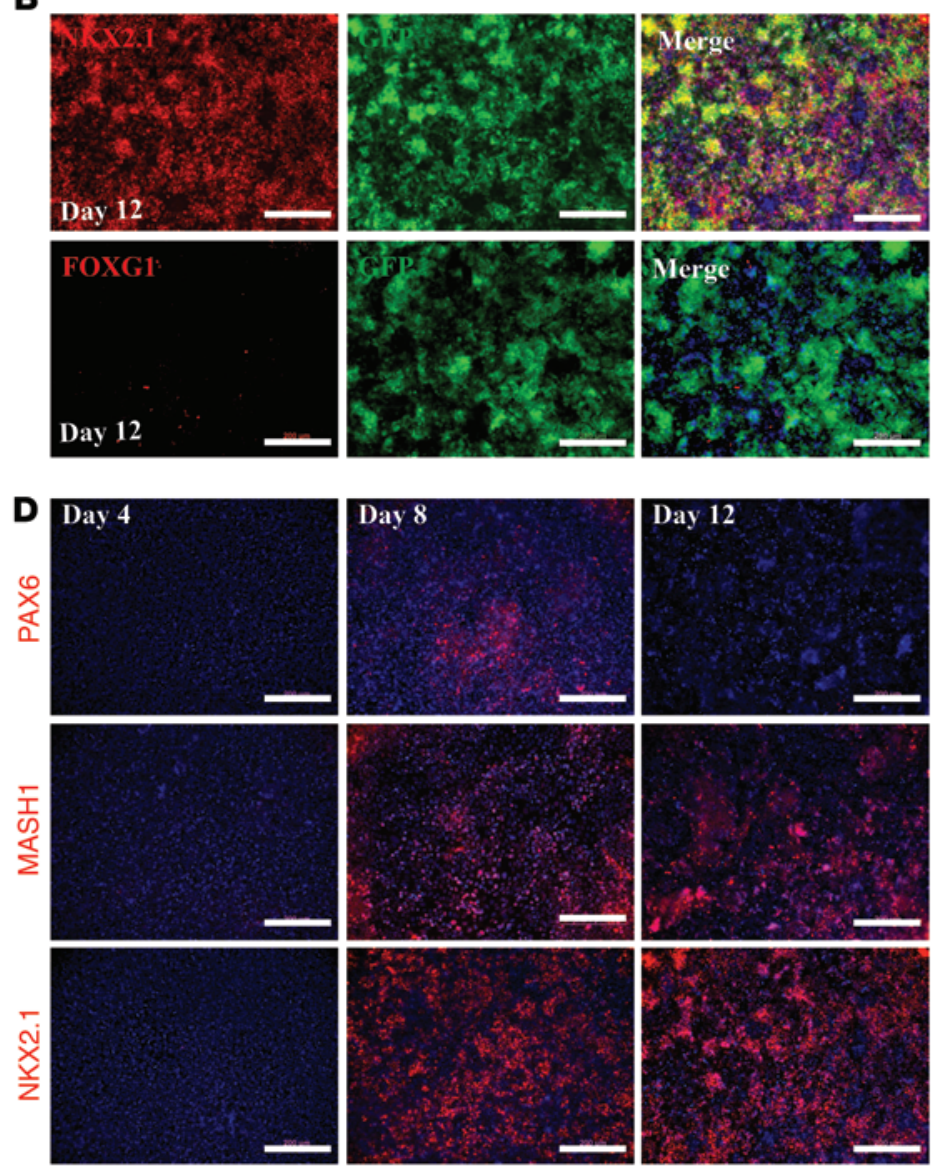

C
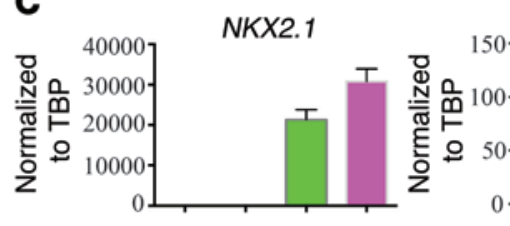

ASCL1/MASH1
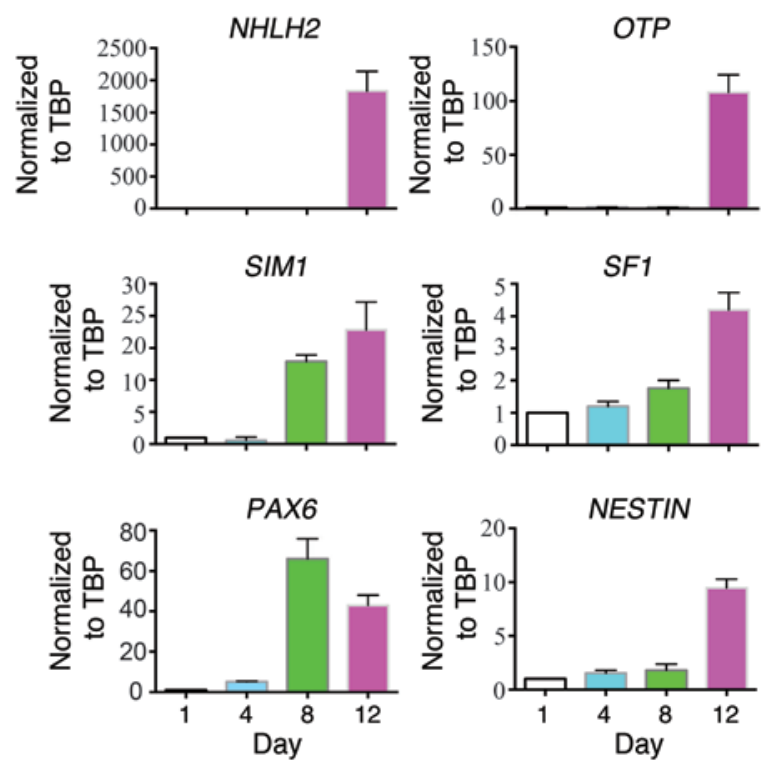

$\mathbf{E}$

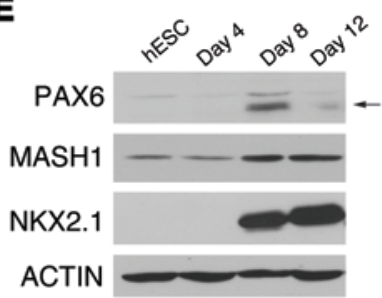

Figure 1. Early activation of SHH signaling and subsequent inhibition of NOTCH signaling induces hypothalamic NKX2.1+ progenitors. (A) Schematic of the differentiation protocol for the generation of hypothalamic progenitors from hESCs/iPSCs. KSR, knockout serum replacement. (B) Immunocytochemical staining of NKX2.1 and FOXG1 in day-12 differentiated cells. Scale bars: $200 \mu \mathrm{m}$. (C) qPCR analysis of indicated TFs on day 1, day 4, day 8, and day 12 of differentiation. $n=3$ for each bar. TBP, TATA box-binding protein. (D) Immunostaining for PAX6, MASH1, and NKX2.1 on days 4, 8, and 12 of differentiation. Nuclei were stained with Hoechst. (E) Western blot analysis for indicated TFs in hESCs after 4, 8, and 12 days of differentiation. Arrow indicates the PAX6 band.

DAPT $(10 \mu \mathrm{M})$ from days 13 to 16 (26). To promote neuron differentiation and survival, cells were cultured in N2-containing medium with brain-derived neurotrophic factor $(\mathrm{BDNF})$ added for a minimum of 8 days starting at day 16 (Figure 2A). POMC and NPY/AGRP neurons are the 2 major neuronal cell types found in the ARC; other neuronal cell types in the ARC include GHRH, somatostatin (SST), and DA neurons (Supplemental Figure 1A). $\alpha \mathrm{MSH}$ and $\mathrm{BEP}$ are among the bioactive products of the processing of POMC propeptide. Both are used as markers for POMC neurons.

In day-24 differentiated cells, we found a mixed hypothalamic neuronal population, including POMC (Figure 2B), NPY (Figure 2C), AGRP (Figure 2D), SST (Figure 2E), and tyrosine hydroxylase-positive (TH-positive) DA (Figure 2F) neurons. These represent most of the cell types identified in the adult ARC (1). NKX2.1 continued to be expressed in these differentiated cells at day 24 (Supplemental Figure 3A). Both POMC and NPY (Figure 2C) neurons expressed GABA (Supplemental Figure 3B) and glutamate decarboxylase 67 (GAD67) (Supplemental Figure 3C). In addition, we also identified a few melanin-concentrating hormone $(\mathrm{MCH})$ $(\sim 1 \%)$ neurons at a late stage (day 65) (Figure 2G). In day-33 neurons, $18 \%$ of cells expressed $\alpha \mathrm{MSH}^{+}$or SST ${ }^{+}$and over $60 \%$ of cells expressed $\mathrm{NPY}^{+}$(Figure 2H).

During mouse hypothalamic development, POMC is transiently expressed in a subset of immature hypothalamic neurons that subsequently develop into other neuronal cell types, such as NPY neurons, in the adult (29). We therefore determined whether similar developmental intermediates occurred during in vitro hESC hypothalamic neuron differentiation. We stained day-29 
A

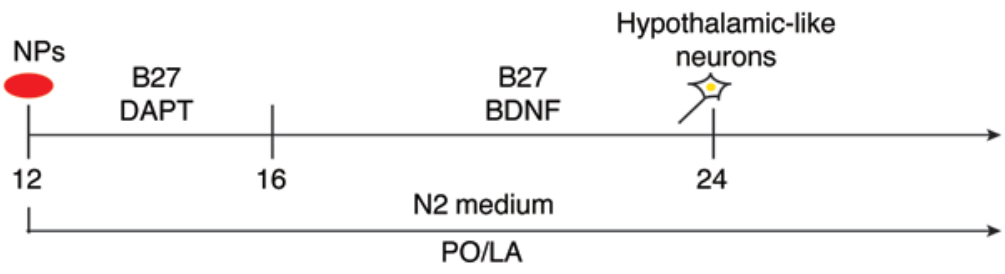

B

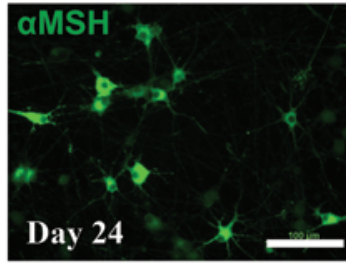

C

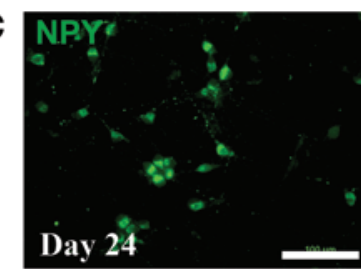

D

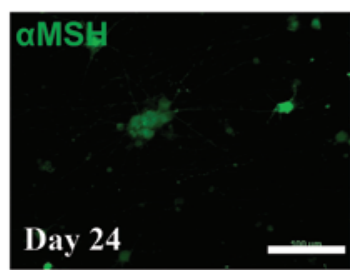

I

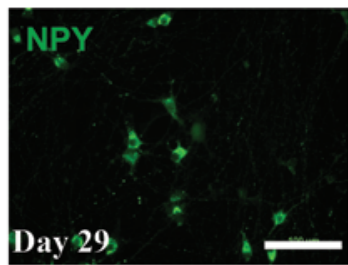

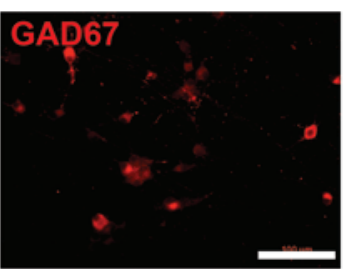
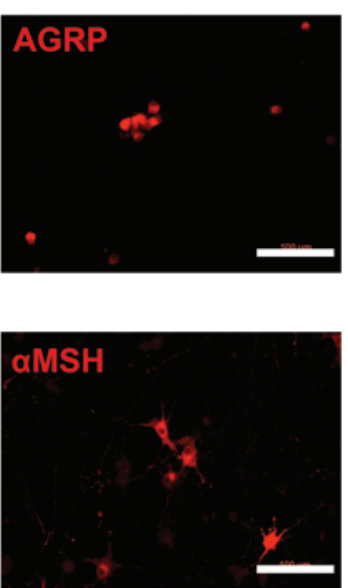

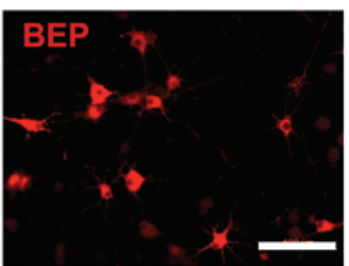

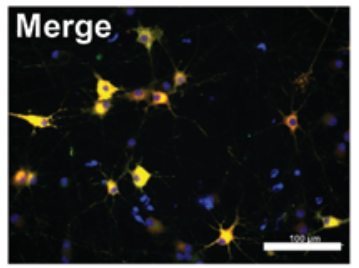
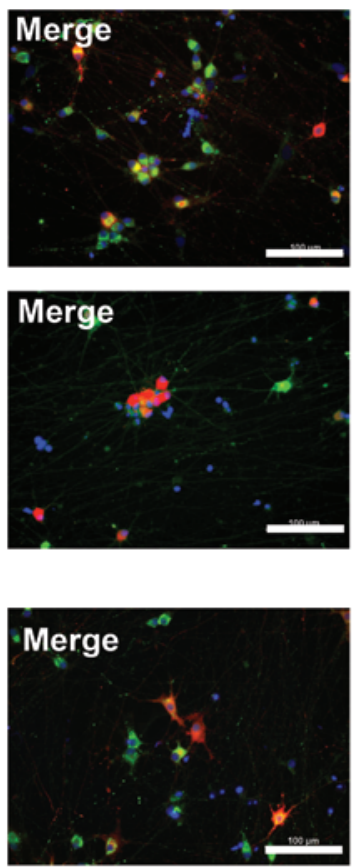

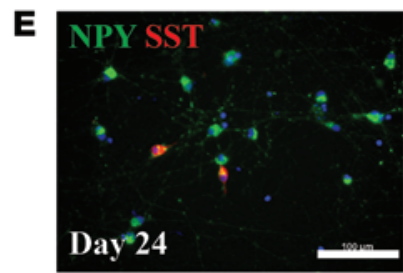

$\mathbf{F}$

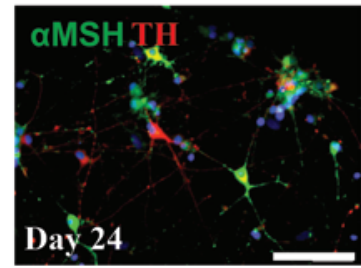

G

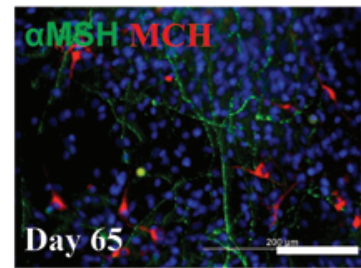

H
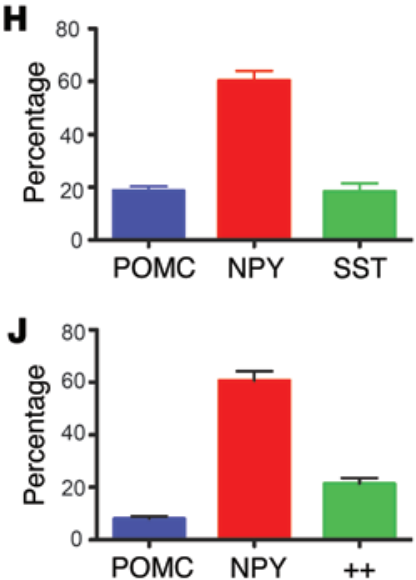

Figure 2. Neurons derived from NKX2.1+FoxG1- progenitors recapitulate hypothalamic ARC neuron identities. (A) Schematic of the differentiation protocol for generating hypothalamic neurons from day-12 neuronal progenitors (continued from Figure 1A). PO/LA, poly-L-ornithine and laminin. (B-G) Immunocytochemical analysis for indicated hypothalamic neuron markers: $\alpha$ MSH, BEP, NPY, AGRP, GAD67, SST, TH, and MCH. Days of differentiation are indicated. (H) Quantification of the percentage of indicated hypothalamic neuron subtypes in day-33 neurons. $n=5$ wells for each bar. (I) Immunocytochemistry of $\alpha \mathrm{MSH}$ and NPY in day-29 neurons. (J) Quantification of neurons that are single- or double-positive for indicated neuropeptides on day 29. $n=5$ wells for each bar. Scale bars: $100 \mu \mathrm{m}$.

neurons for both POMC ( $\alpha \mathrm{MSH})$ and NPY neuropeptides and found that $8 \%$ of neurons expressed only POMC, $61 \%$ of neurons expressed only NPY, and $21 \%$ of neurons were double-positive for POMC and NPY (Figure 2, I and J). This similarity is reminiscent of in vivo mouse hypothalamic ARC neuron development at E13.5-E18.5, at which time POMC and NPY are coexpressed within the same cells (29). In total, neurons expressing primarily POMC or NPY represent $80 \%$ to $95 \%$ of all differentiated cells (based on 3 independent experiments). Thus, inhibition of NOTCH signaling promotes the efficient differentiation of hypothalamic ARClike neurons from NKX2.1 $1^{+}$progenitors.

To determine whether neuropeptide-expressing cells possess the basic functional characteristics of active neurons, their electrophysiological properties were assessed using conventional whole- cell current-clamp recording techniques (30). The subtypes of recorded neurons were identified using post hoc immunostaining for $\alpha \mathrm{MSH}$ and NPY. In day-24 to day-33 cultures, recordings were obtained from 3 types of neurons: $\mathrm{POMC}^{+} \mathrm{NPY}^{+}$(Supplemental Figure 4A), $\mathrm{POMC}^{-} \mathrm{NPY}^{+}$(Supplemental Figure 4B), and doublenegative neurons (Supplemental Figure 4C). All 3 types of neurons fired trains of action potential in response to a 1-second current application (Supplemental Figure 4D). Therefore, hESC-derived hypothalamic neurons have the fundamental electrophysiological properties of neurons.

Transcription profiles confirm a hypothalamic neuron signature. To further characterize transcriptional profiles during hypothalamic neuron differentiation, we performed RNA sequencing (RNA-seq) of undifferentiated hESCs and cells at 12, 27, and 
A
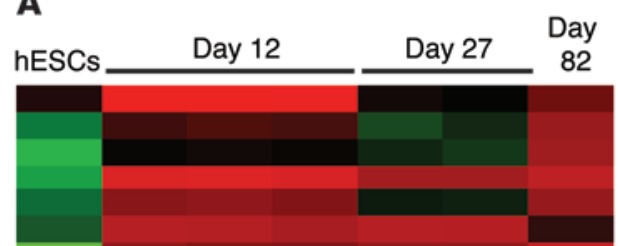

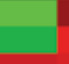
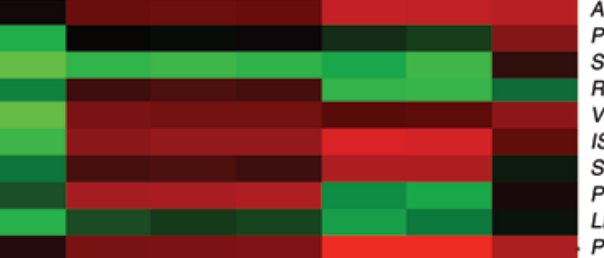

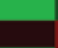

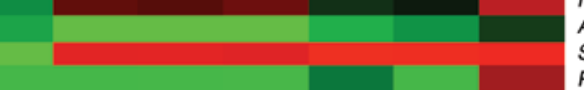

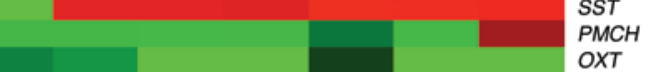
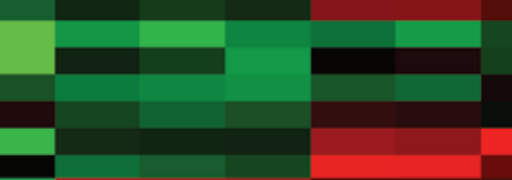

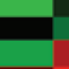
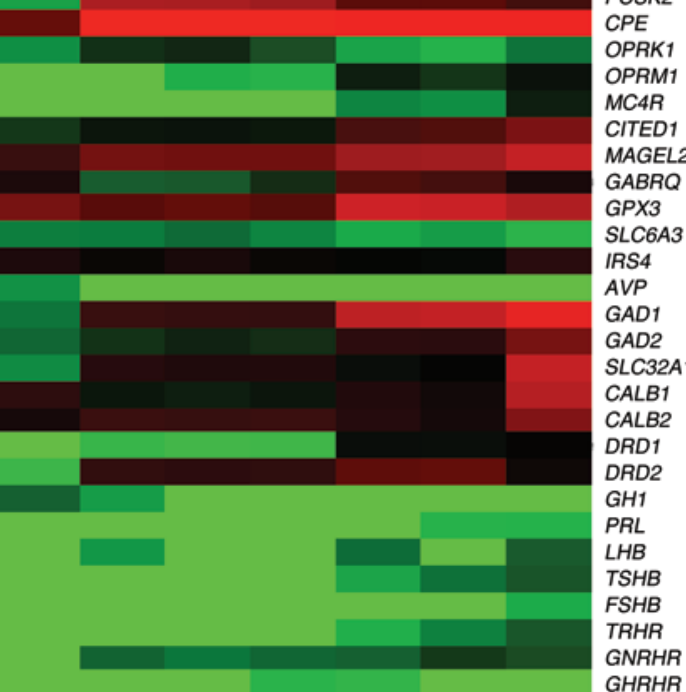

C
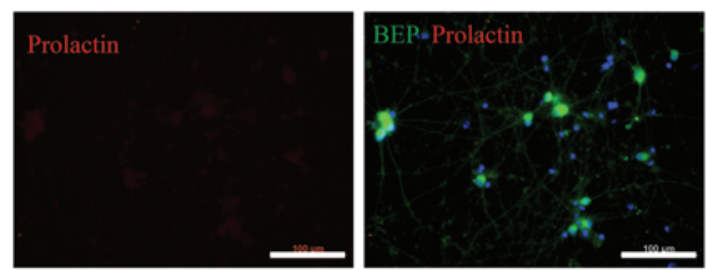

SF1

ARNT2

SIM1

RAX

ISL1

SOX14

PITX2

LMX1B

POMC

NPY

OXT

CRH

GNRH

GNRH1

BDNF

PCSK1

PCSK2

CPE

OPRK1

OPRM1
MC4R

CITED1

MAGEL2

GABRQ

GPX3

SLC6A3

AVP

GAD1

GAD2

SLC32A

CALB1

DRD1

HB

(

SHB

TRHR
GNRHR

GHRHR
B
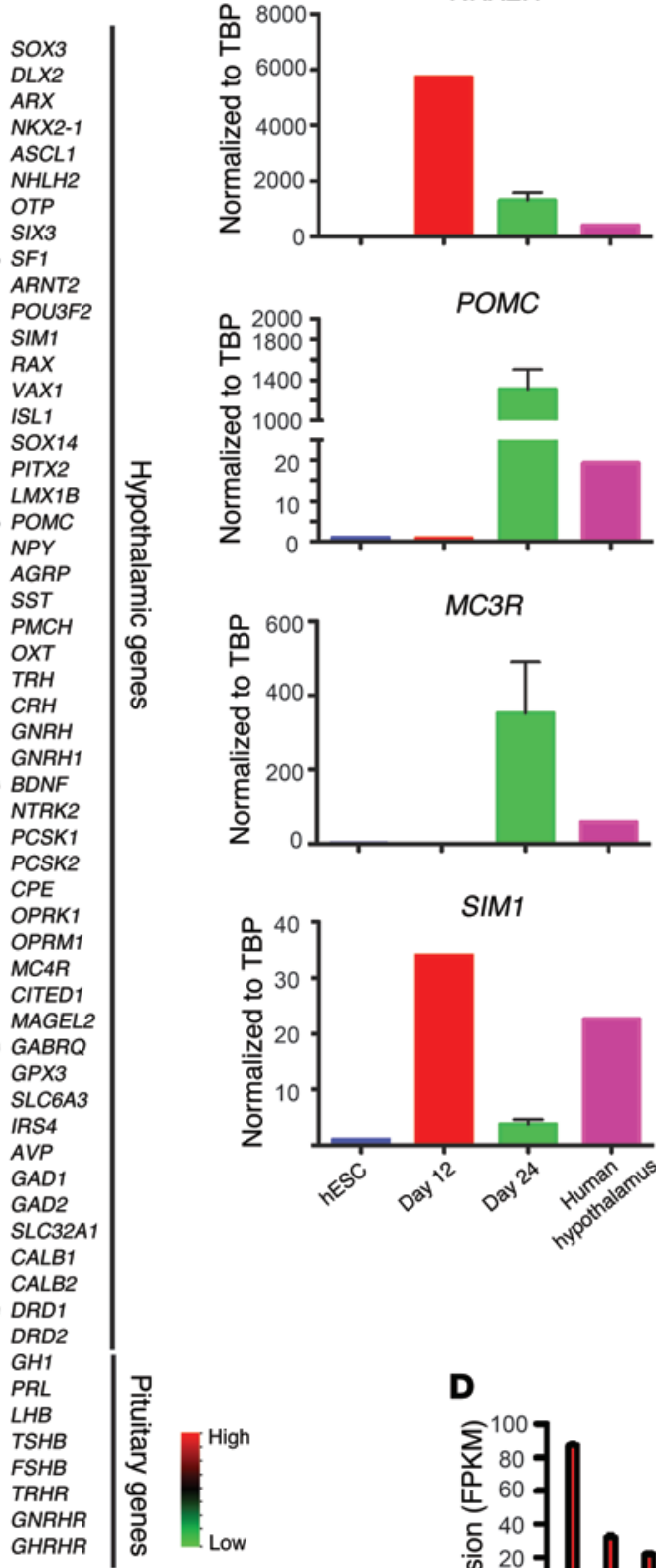

D

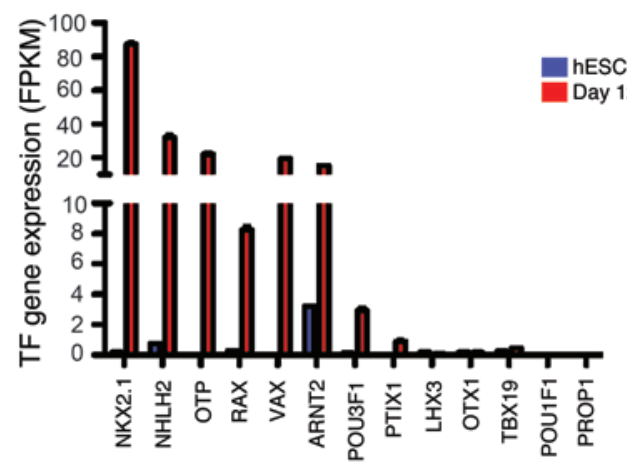

Hypothalamic

Pituitary

Figure 3. In vitro hESC-differentiated neurons show hypothalamic-specific gene signatures. (A) Heat map of 1 matrix clustering of hypothalamic- and pituitary-specific transcripts in undifferentiated hESCs on indicated days of differentiation. Green and red indicate low and high expression, respectively. (B) qPCR analysis of indicated factors in hESCs and of day-12- and day-24-differentiated cells $(n=3)$ and adult human hypothalamus. (C) Immunohistochemistry for prolactin and BEP in day-33 hESC-derived neurons. (D) Gene expression of hypothalamic and pituitary TFs from RNA-seq of hESCs ( $n=1$ ) and day-12 progenitors $(n=3)$. 
82 days of differentiation (protocol in Figure 1A and Figure 2A). Based on the heat maps generated by hierarchical clustering of these transcripts (31) and visualized in Treeview (32), the transcriptional profiles were distinct at these developmental stages (Supplemental Figure 5A). Clusters 6 and 7, which were enriched in day-27 and day-82 differentiated neurons, contained genes participating in neurogenesis, axonogenesis, and neuron function. More specifically, in a pathway analysis of cluster 6 (and similarly for cluster 7), the top hits included opioid POMC, GnRH, oxytocin, $5 \mathrm{HT} 2$ receptor, histamine $\mathrm{H} 1 / 2$ receptor, and circadian clock system signaling, which participate in hypothalamus-specific functions (Supplemental Figure 5A and ref. 1).

We also generated heat maps for neuronal and hypothalamicspecific transcripts. TFs NKX2.1, ASCL1, NHLH2, OTP, SIX3, VAX, $S O X 3, R A X, S F 1$, and ARNT2, which participate in hypothalamic neuron differentiation, were enriched in day-12 cells (Figure $3 \mathrm{~A}$ ). Most hypothalamic neuron-specific transcripts, such as $P O M C$, $S S T, T R H$, and carboxypeptidase E (CPE) (enzyme required for POMC processing), were highly expressed in day-27 and day- 82 differentiated neurons, were expressed at low levels in neural progenitors, and were not expressed in hESCs (Figure 3A). The GABAergic neuron-specific transcripts (GAD1, GAD2, SLC32A1, CALB1, and $C A L B 2)$ were also enriched in these differentiated hypothalamic neurons, consistent with our previous finding that most of these neurons are ARC like. The persistent expression of hypothalamicspecific genes in day-27 and day- 82 neurons indicated that the hypothalamic identity of these in vitro-differentiated neurons was stable for at least 2 months. In addition, day- 12 progenitors could be kept frozen at $-80^{\circ} \mathrm{C}$ for at least 11 months and, when thawed, retained the ability to differentiate into hypothalamic neurons.

To confirm the RNA-seq findings, we performed qPCR analysis of TFs required for hypothalamic neuron differentiation and for hypothalamus-specific transcripts in hESC and cells from 12, $18,24,36$, and 40 days of differentiation (Supplemental Figure $5 \mathrm{~B}$ ) and compared gene expression in day-24 neurons with that of adult human hypothalamus RNA (Figure 3B and ref. 1). All of the hypothalamic genes tested showed a time-dependent upregulation across the differentiation period examined (Supplemental Figure 5B). Compared with adult human hypothalamus, the transcript levels of NKX2.1, OTP, POMC, and MC3R in day-24 differentiated neurons were higher, while transcripts of other genes (AGRP, LEPR, SF1, and SIM1) were comparable to or lower than those in adult human hypothalamus (Figure $3 \mathrm{~B}$ ). Among these genes, the POMC transcript level was about 60-fold higher than that in adult human hypothalamus, suggesting that POMC neurons were highly enriched in these hESC-derived neurons. "Dilution" of POMC neurons by other non-ARC neurons and astrocytes in the adult human hypothalamus presumably accounts for the relative elevation of POMC expression in the hESC-derived neurons.

The presence of hypothalamic transcripts alone is insufficient to confirm hypothalamic identity. The hypothalamus and pituitary share several developmental transcriptional factors, such as PITX1, PITX2, LHX4, and SF1 $(33,34)$, and POMC-expressing neurons are also present in the anterior pituitary, which produces adrenocorticotropic hormone $(\mathrm{ACTH})$ to regulate the production and release of adrenal corticosteroids (35). However, based upon RNA-seq, none of the pituitary-specific transcripts - GH1, PRL,
LHB, TSHB, FSHB, TRHR, GNRHR, GHRHR - were expressed in differentiated neurons (Figure $3 \mathrm{~A}$ and ref. 34). Also by immunocytochemistry, prolactin was not detectable in differentiated neurons (Figure 3C). And finally, in day-12 progenitors, none of the pituitary TFs (PITX1, LHX3, OTX1, TBX19, POU1F1, or PROP1) were expressed in these cells (Figure 3D), whereas hypothalamic TFs (NKX2.1, NHLH2, OTP, RAX, VAX, ARNT2, and POU3F1) were highly expressed. In summary, differentiated neurons displayed hypothalamic, but not pituitary, specific transcript signatures, demonstrating that these were not pituitary cell types.

hESC-derived neurons produce and secrete hypothalamic neuropeptides. In the hypothalamus, POMC propeptide undergoes proteolytic processing by prohormone convertases 1 (PC1), 2 (PC2) and $\mathrm{CPE}$ to generate bioactive short peptides, including $\mathrm{ACTH}$, $\alpha \mathrm{MSH}$, and BEP (36). mRNAs for PCSK1, PCSK2, and CPE were increased in day-24 hypothalamic neurons compared with hESC day-12 progenitors and were as highly expressed as in the human adult hypothalamus (Figure 4A). We further found that CPE was colocalized with $\alpha-\mathrm{MSH}$ in POMC neurons (Figure 4B). Large amounts of POMC, $\alpha \mathrm{MSH}$, and BEP protein were detected in lysates of neuronal cells at 24 and 45 days of differentiation (Figure $4 \mathrm{C}$ and ref. 37 ). Day- 45 neurons produced more POMC and processed neuropeptides ( $\alpha \mathrm{MSH}$ and BEP) than day-24 neurons. The increased processing of POMC peptides in these neurons was reflected by the higher ratios of $\alpha \mathrm{MSH} / \mathrm{POMC}$ and $\mathrm{BEP} /$ POMC on day 45 compared with day 29 (Figure 4D). A similar increase in processing of neuropeptides is seen in the developing mouse hypothalamus (38).

Culture medium of day- 45 neurons contained high levels of POMC, ACTH, $\alpha \mathrm{MSH}$, and BEP, suggesting that these neurons secreted these neuropeptides (Figure 4E). AMSH and BEP proteins are stored in secretory granules in POMC neurons, which undergo exocytosis to release these neuropeptides in response to appropriate stimuli (35). To determine whether neurons had the ability to secrete neuropeptides in response to membrane depolarization and to exclude cell death as a source of the peptides in the culture medium, we treated day- 40 neurons with $60 \mathrm{mM} \mathrm{KCl}$ for 20 minutes and measured concentrations of neuropeptides in the medium. $\mathrm{KCl}$ stimulated the secretion of both $\alpha \mathrm{MSH}$ and BEP by more than 2-fold (Figure 4F). Unprocessed POMC was also detected in neuronal lysates and in the culture medium, but showed only a slight increase in response to $\mathrm{KCl}$. Thus, selective secretion of processed neuropeptides was responsible for the changes in media concentrations of $\alpha \mathrm{MSH}$ and BEP.

In addition to POMC peptides, we further asked whether these neurons could produce other hypothalamic-specific neuropeptides, such as AGRP. AGRP was detectable by radioimmunoassay in neuron lysates from early differentiated neurons (day 29), and increased about 4 -fold by day 45 (Figure $4 \mathrm{G}$ ), consistent with the increase in $A G R P$ transcripts (Supplemental Figure 5B). Moreover, receptors for $\alpha \mathrm{MSH}(M C 2 R, M C 3 R$ [Figure 3B], $M C 4 R)$ and opioid receptors for BEP (OPRM1 and OPRD1) (39) were highly expressed in day-24 neurons, consistent with the likelihood that some of these neurons represent "downstream" members (e.g., PVN) of the melanocortin-signaling pathway (Figure 4H). Therefore, these hESC-derived neurons display key aspects of the neuroendocrine function of hypothalamic ARC-like neurons. 
A

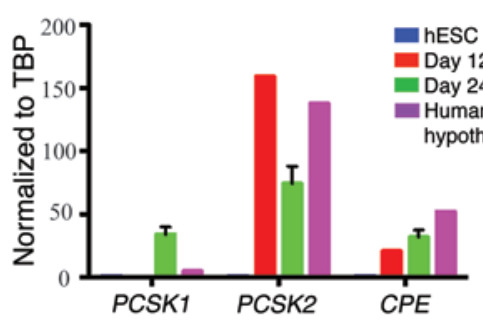

C

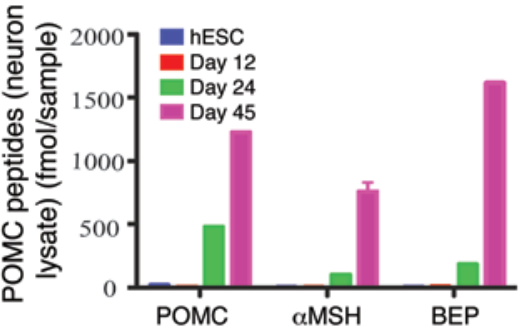

$\mathbf{F}$

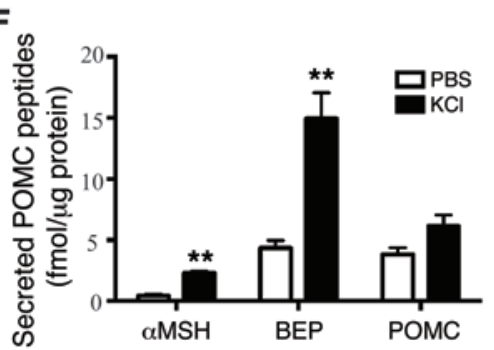

B

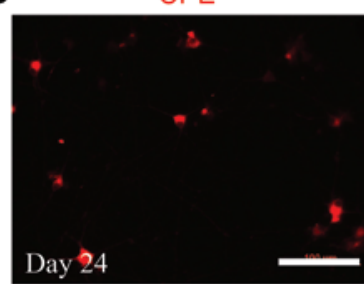

$\alpha \mathrm{MSH}$

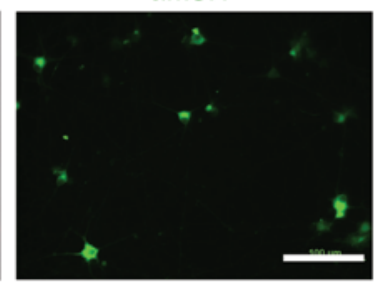

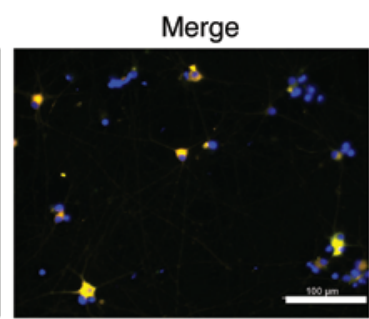

D

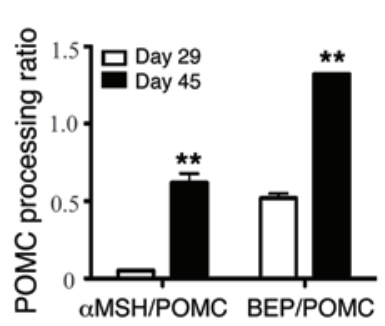

E

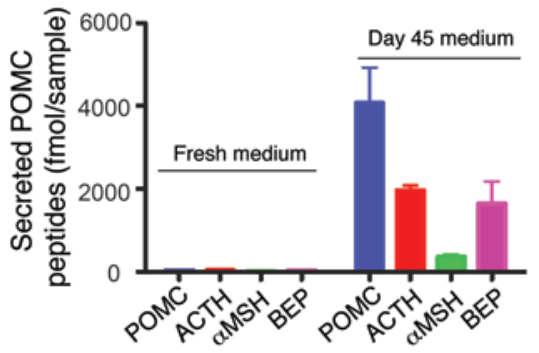

G

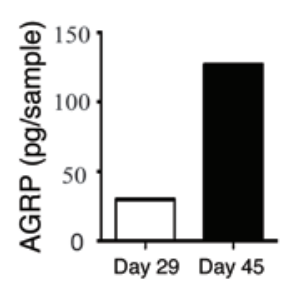

H

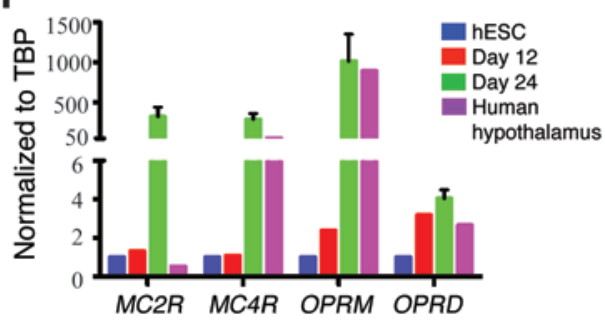

Figure 4. hESC-derived hypothalamic neurons produce and secrete neuropeptides related to ingestive behaviors. (A) qPCR analysis of gene expression of prohormone convertases as indicated in hESCs, day-12 progenitor, and day-24 hESC-derived hypothalamic neurons. Human hypothalamus RNA was used for comparison. (B) Immunocytochemistry of CPE and $\alpha$ MSH in day-24 hESC-derived neurons. Scale bars: $100 \mu \mathrm{m}$. (C) ELISA assay for POMC neuropeptide; RIA for $\alpha$ MSH and BEP in the lysate of hESCs, 12, $24(n=1)$, and $45(n=3)$ days of differentiation. (D) Ratios of $\alpha$ MSH/POMC and BEP/POMC in day-24 and day-45 neurons; $n=3-4$ for each bar. (E) The amount of POMC, ACTH, $\alpha M S H$, and BEP in day-45 neuron culture medium. (F) The influence of KCl on $\alpha M S H$, BEP, and POMC secretion in day-40 neurons. $n=3$ for each bar. (G) RIA of AGRP production in day- 29 and day-45 neurons $(n=1)$. (H) qPCR analysis of gene expression of melanocortin receptors and receptors for BEP as indicated in the same samples as shown in $\mathbf{A} .{ }^{* *} P<0.01,2$-tailed Student's $t$ test.

NKX2.1 ${ }^{G F P / W}$ hESC-derived neurons can sense and respond to insulin and leptin. Critical to the role of the hypothalamus in energy homeostasis is the ability of POMC- and NPY/AGRPexpressing neurons in the ARC nucleus to sense and respond to circulating hormones such as insulin and leptin and to release neuropeptides ( $\alpha \mathrm{MSH}, \mathrm{NPY}$, and AGRP) that further activate downstream MC4R-mediated melanocortin signaling in VMN and PVN neurons (3). Therefore, we determined whether hESCderived hypothalamic ARC-like neurons could sense and respond to metabolic hormones.

We found that the insulin receptor gene, INSR, was expressed in differentiated day-25 to day-27 neurons (Supplemental Figure $6 \mathrm{~A}$ ). In addition, the insulin signaling molecules IRS2 (Supplemental Figure 6B) and IRS4 (Supplemental Figure 6C) were also expressed in these cells. To test their ability to respond to insulin, day-33 differentiated neurons were starved in neurobasal medium plus B27 (minus insulin) for 16 hours and then exposed to $1 \mu \mathrm{g} / \mathrm{ml}$ insulin for 30 minutes at $37^{\circ} \mathrm{C}$. Using Western blot analysis, we detected a robust induction of AKT phosphorylation (on Thr308 and Ser473) after insulin administration (Figure 5A). An inhibitor of AKT (AKTi) reduced the production of POMC and BEP in day-
38 neurons (Figure 5B), indicating that neuropeptide production in these neurons might be regulated by metabolic signals (40-42).

To assess leptin signaling, we quantified expression of the leptin receptor (LEPR) and examined phosphorylation of STAT3, a downstream signal transducer of leptin signaling (43).

LEPR mRNA was first detected on day 18 of differentiation; levels were approximately $60 \%$ of those in adult human whole hypothalamus on day 24 of differentiation (Figure 3B) and further increased in day-36 and day-40 neurons (Figure 5C). When day-34 neurons were exposed to leptin $(1 \mu \mathrm{g} / \mathrm{ml})$, Western blot analysis revealed a 2-fold increase in p-STAT3 levels (Figure 5D). However, leptin-treated neurons showed no increase of SOCS3 (downstream of LEPR) expression (Supplemental Figure 6D), and the number of p-STAT3 immunopositive day-29 neurons was less than $1 \%$ following leptin treatment (Supplemental Figure 6E).

We reasoned that the functional immaturity of these neurons might be improved by longer culture and by coculture with astrocytes (44). Accordingly, we cultured these hESC-differentiated cells with mouse cortical astrocytes from day 12 to day 53 of differentiation and then exposed the neurons to leptin. With leptin treatment, we noted an increase in p-STAT3-positive cells (from $<1 \%$ 
A

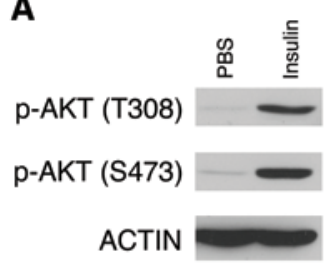

D

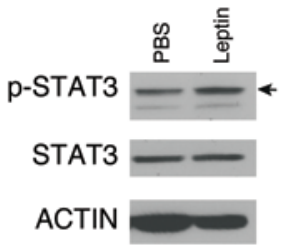

B

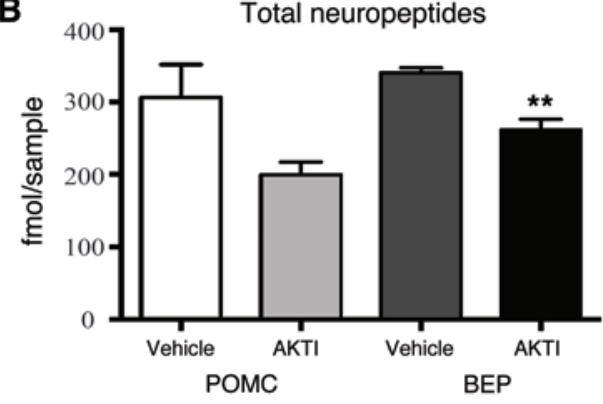

C

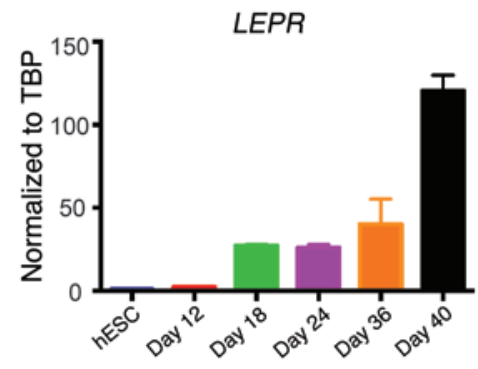

E

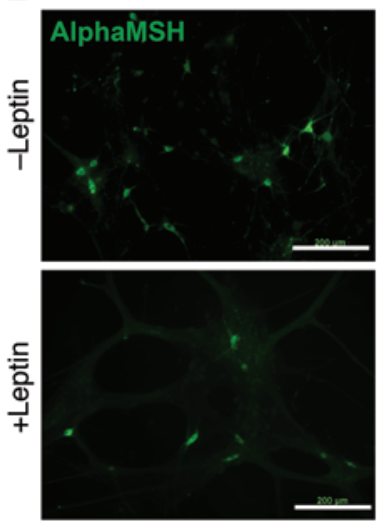

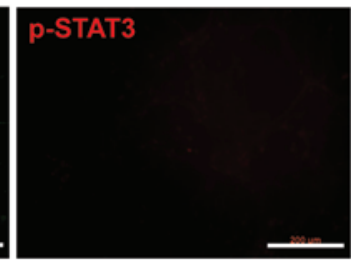

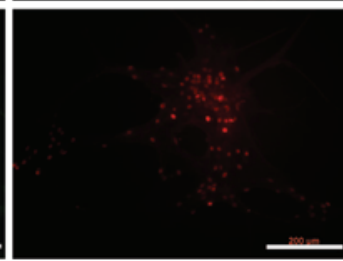

G

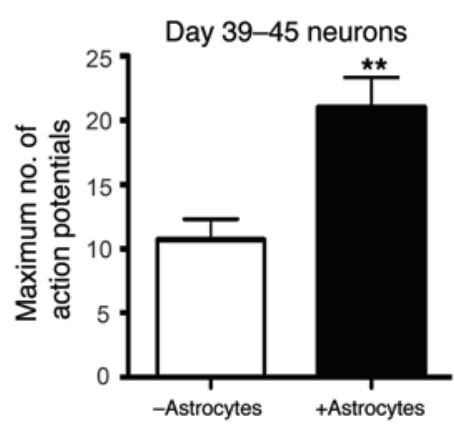

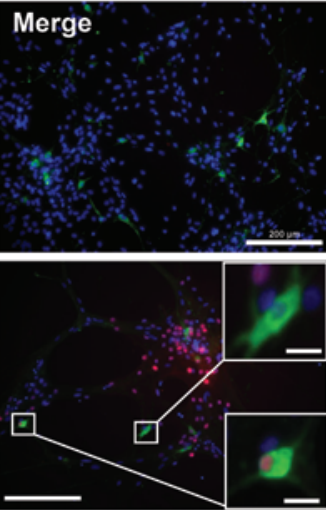

H

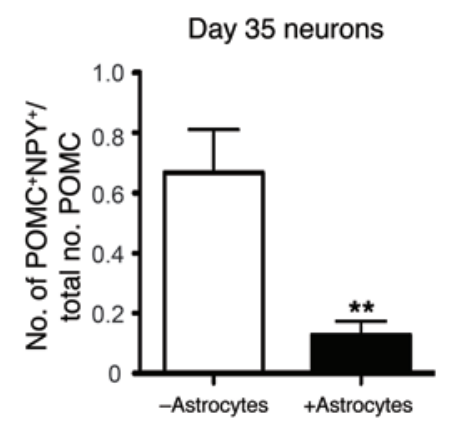

Figure 5. hESC-derived hypothalamic ARC-like neurons sense metabolic signals insulin and leptin. (A) Western blot analysis of insulin-treated ( $1 \mu \mathrm{g} /$ ml) day-33 hESC-derived hypothalamic neurons. Antibodies for p-AKT (Thr308) and p-AKT (Ser473) were probed. (B) Inhibition of insulin signaling by AKTi decreases the production of neuropeptides in day-38 hESC-derived POMC neurons. Total neuropeptide is the sum of the quantities of neuropeptides in the medium and neural lysates. $n=3$ for each bar. (C) Transcript levels of LEPR in hESCs and 12, 18, 24, 36, and 40 days of differentiation ( $n=3$ ). (D) Western blot analysis of leptin-treated $(1 \mu \mathrm{g} / \mathrm{ml}$ ) day-33 hESC-derived hypothalamic neurons. p-STAT3 and STAT3 were probed. (E) Immunocytochemistry for p-STAT3 and $\alpha \mathrm{MSH}$ in day- $53 \mathrm{hESC}$-derived hypothalamic neurons cocultured with mouse cortical astrocytes. Rectangle indicates 2 subtypes of POMC neurons: leptin sensing and nonsensing. Scale bars: $200 \mu \mathrm{m} ; 25 \mu \mathrm{m}$ (small panels). (F) Action potential firing of neurons with or without mouse astrocyte coculture. Dashed lines indicate $-60 \mathrm{mV}$. (G) Quantification of the maximal number of action potentials in neurons cocultured with mouse cortical astrocytes $(n=6)$ and neurons cultured without astrocytes $(n=10)$. (H) Quantification of the ratios for POMC+ NPY+ in day-35 neurons with or without astrocyte coculture. The ratio is calculated with the following equation: (number of $\mathrm{POMC}^{+} \mathrm{NPY}^{+}$)/(total number of POMC neurons). $n=3-5 .{ }^{* *} P<0.01$, 2-tailed Student's $t$ test.

to $>20 \%$ ) among these cocultured neurons, including p-STAT3POMC-coexpressing neurons (Figure 5E). This improvement was further supported by c-Fos staining, another downstream indicator of leptin signaling (Supplemental Figure 6F). Other indications of enhanced neuronal maturation in the presence of mouse astrocytes were an increase in the maximal number of action potentials (Figure 5, F and G) and a reduction in the percentage of POMC/ NPY-coexpressing cells (versus the total number of POMC neurons) in day-35 neurons (Figure 5H and Supplemental Figure 6G). Therefore, hESC-derived hypothalamic cells are responsive to metabolic signals such as insulin and leptin, and functional maturation of these cells is improved by astrocyte coculture.

Differentiation of hypothalamic ARC-like neurons from iPSCs. One important use of the cells described here will be in understanding the molecular physiology of hypothalamic control of human body weight. We therefore generated iPSC lines from 3 subjects with BBS (45) and 2 healthy controls. These iPSC lines showed the expected characteristics of human pluripotent stem cells and a diploid karyotype (Supplemental Figure 7). Using the protocol established for the hESC line, on average, $80 \%$ of 
A

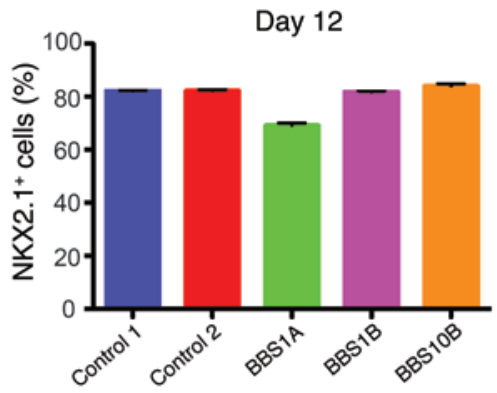

C

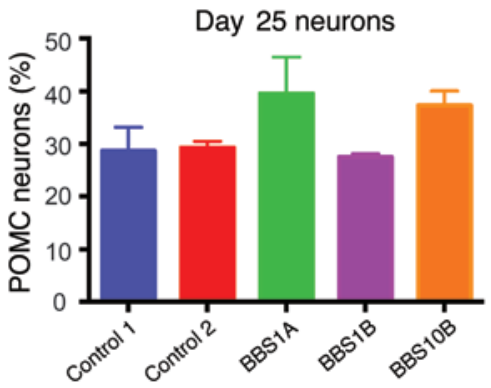

E

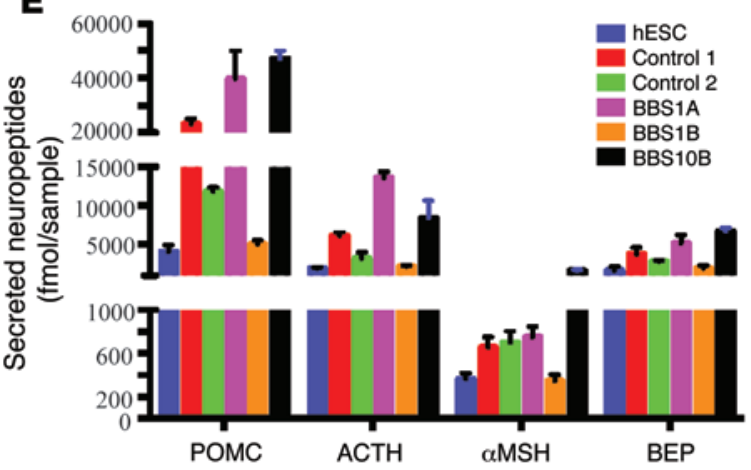

$\mathbf{F}$

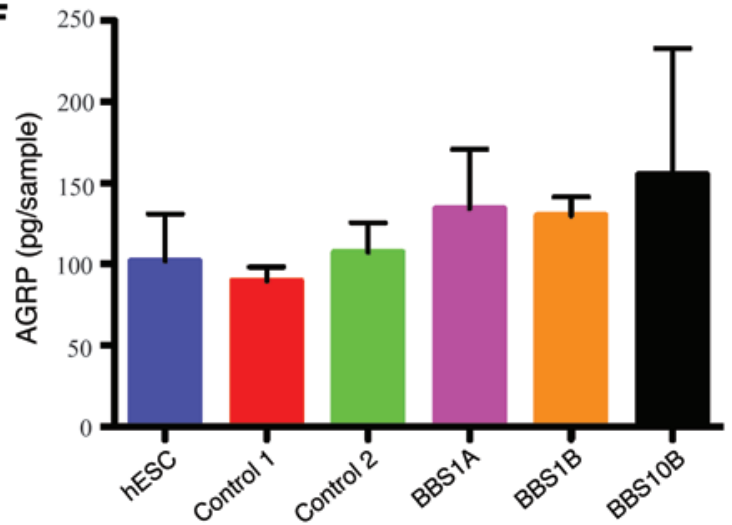

B

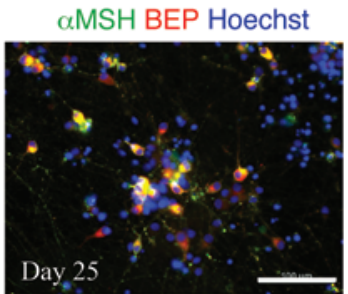

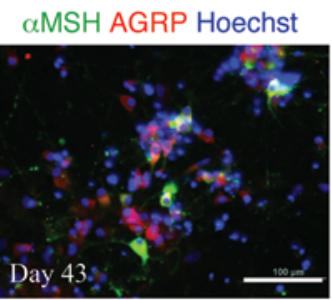

aMSH NPY Hoechst

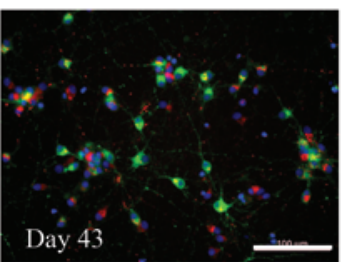

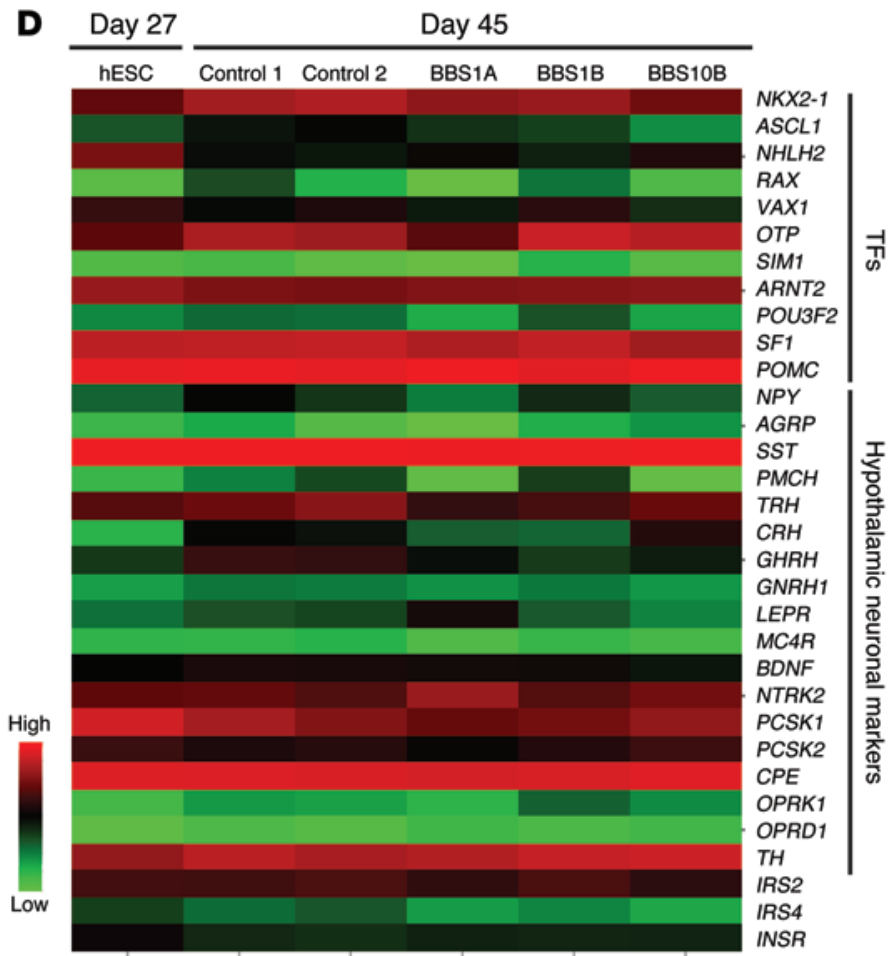

G

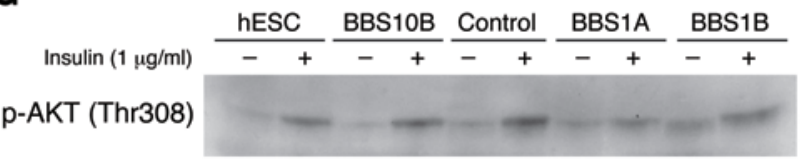

p-AKT (Ser473)

AKT

Figure 6. Efficient generation of hypothalamic ARC-like neurons from iPSCs generated from BBS obese subjects. (A) Quantification of the percentage of NKX2.1 cells in day-12 progenitors derived from 2 control iPSC lines (control 1, control 2 ) and 3 BBS iPSC lines (BBS1A, BBS1B, BBS10B). $n=5$ wells for each bar. (B) Immunostaining of day-25 and day-43 iPSC-derived neurons. Scale bars: $100 \mu \mathrm{m}$. (C) Quantification of the percentage of POMC neurons $(n=3$ to 5 wells). (D) Heat map of the expression of hypothalamic TFs and neuronal markers in day-27 hESC-derived and day-45 iPSC-derived neurons based on the FPKM value from RNA-seq. Red color indicates high expression; green color indicates low expression. Action potentials in day-30 control iPS-derived and day-35 BBS iPS-derived neurons. (E and F) Quantification of secreted neuropeptides as indicated on day 45 of differentiation. The volume of each sample was $1 \mathrm{ml} . n=3$ for each bar. (C) Western blot analysis of insulin signaling in day-34 hESC-derived and in iPSC-derived neurons. 
NKX2 $21^{+}$progenitors were obtained from all 5 independent iPSC lines (Figure 6A and Supplemental Figure 8A). The iPSC-derived NKX2.1 progenitors could be further differentiated into hypothalamic ARC-like neurons, including POMC, NPY, and AGRP neurons (Figure 6B and Supplemental Figure $8 \mathrm{~B}$ ). The percentage of POMC neurons ranged from $27.5 \%$ to $39.6 \%$ in all 5 iPSC lines (Figure 6C), comparable to the rates obtained in the hESC-derived ARC neurons. RNA-seq of day-45 iPSC-derived neurons showed patterns of hypothalamic and neuronal markers virtually identical to those obtained in the hESC-derived cells (Figure 6D).

To assess the functions of these iPSC-derived neurons, we first obtained current-clamp recordings on these cells. These cells displayed action potentials, further confirming their neuronal identity (Supplemental Figure 8C). With regard to neuropeptide production and secretion, these iPSC-derived neurons produced amounts of POMC, ACTH, $\alpha \mathrm{MSH}, \mathrm{BEP}$, and AGRP comparable to those of hESC-derived neurons (Figure 6, E and F). By RNA-seq, levels of LEPR and the insulin-signaling molecules INSR, IRS2, and IRS4 were comparable between hESC- and iPSC-derived neurons (Figure 6D). Insulin induced a 5- to 10-fold increase in levels of p-AKT in these iPSC-derived neurons (Figure 6G), suggesting these neurons could also respond to metabolic signals. These data indicate comparable functionality between hESC- and iPSC-derived hypothalamic neurons and suggest the suitability of the latter for pathogenetic studies of patient-specific cells.

\section{Discussion}

We developed a protocol for the efficient and reproducible generation of hypothalamic ARC-like neurons from both human ESCs and patient-specific iPSCs. Early activation of SHH signaling and dual SMAD inhibition (days 1 to 8) followed by inhibition of Notch signaling (days 9 to 12) drives human pluripotent stem cells toward ventral diencephalon progenitors with the transcriptional signature $\mathrm{NKX} 2.1^{+} \mathrm{FOXG1}^{-}$. With further differentiation in the presence of the Notch inhibitor DAPT (days 13 to 16) and neurotrophic factor BDNF (day 17 to the end of differentiation), neurons differentiated from the $\mathrm{NKX}^{2} .1^{+} \mathrm{FOXG}^{-}$neural precursors are hypothalamic in nature because (a) these cells are derived from the ventral NKX2.1 progenitors, but not telencephalon MGE progenitors, and the NKX2.1 progenitors express multiple TFs, such as ASCL1, NHLH2, OTP, SIM1, SF1, RAX, and VAX, which are required for the differentiation and specification of hypothalamic neurons. (b) Differentiated neurons at day 24 include high percentages of POMC, NPY/AGRP, SST, and DA neurons, which together account for over $90 \%$ of the cells generated. Most POMC and NPY neurons are also GAD67 or GABA positive, consistent with the in vivo data from rodents (46). Of the day-29 differentiated neurons, POMC- and/or NPY-expressing neurons account for $90 \%$ of the cells. These cell types thus recapitulate hypothalamic ARC neuron identities. (c) RNA-seq, qPCR analysis,and immunocytochemistry indicate that the differentiated neurons display hypothalamic gene expression signatures, including $P O M C$, NPY, AGRP, MC3R, GHRH, SIM1, SF1, TRH, PMCH, and CPE. (d) $P O M C$ is also highly expressed in the anterior pituitary. However, we found that the pituitary-specific TFs (PITX1, LHX3, PITX3, OTX1, TBX19, POU1F1 [PIT1], and PROP1) were not expressed in day-12 NKX2.1-GFP precursors, and pituitary-specific transcripts
(GH1, PRL, LHB, TSHB, and FSHB) were not expressed in differentiated neurons. These results indicate that the protocol reported here does not create pituitary cells. (e) The differentiated neurons displayed key functional properties of hypothalamic neurons, including the ability to accurately process and secrete neuropeptides ( $\alpha \mathrm{MSH}, \mathrm{BEP}, \mathrm{AGRP})$ and to respond to metabolic signals such as insulin and leptin.

GWAS and whole-exome sequencing studies continue to identify many candidate genes for human obesity (47-49). The functional analysis of these candidates in rodents is time consuming, costly, and subject to strain-related effects on phenotypes related to energy homeostasis and diabetes (50-52). Additionally, the effects of patient-specific gene $\times$ gene interactions will be informative regarding molecular pathogenesis (53). However, it is impossible to analyze the molecular basis of obesity directly with human neuronal tissue. Alternatively, human pluripotent stem cells are known for their characteristics of self renewal and pluripotency and could provide unlimited cell resources to address such issues. The protocol described here has been reproducible in all hESCs and iPSC lines tested, resulting in more than $80 \%$ to $95 \%$ neurons with hypothalamic characteristics, and should be useful for the routine generation of patient-specific hypothalamic neurons. Furthermore, human pluripotent stem cells can be manipulated genetically to alter DNA sequences in candidate genes identified in human genetic screens and to "correct" mutations in cells from individual obese patients with gene-editing technologies, such as Zinc finger nuclease or CRISPR/Cas9 $(54,55)$. In both instances, the result is the creation of allelic series on a constant genetic background, eliminating the "noise" introduced by differences in such backgrounds between affected and controls. The study of human hypothalamic neurons generated using the protocol described here should be helpful in delineating the molecular pathogenesis of monogenic and other types of human obesity and in assessing the responses of these cells to therapeutic agents.

\section{Methods}

Generation and characterization of iPSCs from human fibroblasts. We obtained skin biopsies from BBS patients and generated fibroblast lines using the same methods described previously (56). Primary fibroblasts were reprogrammed into pluripotent stem cells using retrovirus. 5,000 fibroblast cells were seeded per well in a 12 -well dish. Once fibroblasts attached to the plate, they were infected with retroviruses expressing human TFs OCT4, SOX2, Klf4, and C-Myc (57). The procedure for generating and culturing iPSC lines was the same as described by us earlier (56). hESCs/iPSCs were maintained in hESC medium: $500 \mathrm{ml}$ knockout DMEM, 90 ml knockout serum, $6.5 \mathrm{ml}$ GlutaMAX, $6.5 \mathrm{ml}$ NEAA, $6.5 \mathrm{ml}$ penicillin/streptomycin, $0.65 \mathrm{ml} \beta$-mercaptoethanol, and $10 \mathrm{ng} / \mathrm{ml} \mathrm{bFGF}$. All reagents were from Life Technologies.

Hypothalamic neuron differentiation protocol. Human ESCs/iPSCs were cultured overnight on Matrigel plates (1 million cells/well for 6-well plates) in hESC medium with $10 \mu \mathrm{M}$ ROCK inhibitor (Y27632, Stemgent) and then differentiated in EB medium: hESC medium without bFGF, supplemented with SHH (100 ng/ml, R\&D), PM ( $2 \mu \mathrm{M}$, Stemgent), $10 \mu$ M SB 431542 (Selleckchem), and $2.5 \mu$ M LDN 193289 (Selleckchem). From days 4 to 8, EB medium was gradually replaced with N2 medium (25) (500 ml DMEM/F12 supplemented with 5.5 $\mathrm{ml} \mathrm{N} 2$ supplement, $0.2 \mathrm{mM}$ ascorbic acid, $5.5 \mathrm{ml}$ GlutaMAX, $5.5 \mathrm{ml}$ 


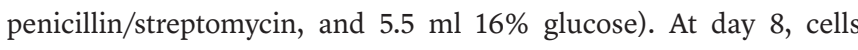
were switched into N2 medium supplemented with B27 and $10 \mu \mathrm{M}$ DAPT (Stemgent) until day 12. Cells were harvested with trypsin LE (Life Technology) at $37^{\circ} \mathrm{C}$ for 4 minutes and washed twice with N2 medium; the pellet was then resuspended with N2 medium plus B27 and $10 \mu \mathrm{M}$ ROCK inhibitor and passed through a Falcon tube with cell-strainer cap (Fisher Scientific) to make a single-cell suspension. Cells were counted and aliquots plated on poly-L-ornithine- $(0.01 \%$, Sigma-Aldrich) and laminin-coated $(4 \mu \mathrm{g} / \mathrm{ml}$, Life Technology) plates (150,000 cells/well for 24-well plate, 300,000 cells/well for 12-well plate, 1 million cells/well for 6-well plate). After cells completely attached to the plate, medium was changed to N2 medium plus B27 and $10 \mu \mathrm{M}$ DAPT. Four days later, cells were switched to N2 medium supplemented with B27 and $20 \mathrm{ng} / \mathrm{ml}$ BDNF (R\&D) until analysis. Medium was changed every 2 days until analysis. The hESC/iPSCderived day-12 hypothalamic progenitors could be cryopreserved for long-term storage (>11 months). The viability of the frozen progenitors after thawing was more than $95 \%$. The thawed cells performed identically to the nonfrozen cells described in these studies.

Primary mouse cortical astrocytes were prepared as described (58). We split these mouse astrocytes once onto poly-L-ornithine and laminin-coated plates with a density of 20,000 to 30,000 cells for a 4 -well plate 1 week before we seeded them onto the day- 12 progenitors. The differentiation protocol for these cocultures was the same as described above.

Electrophysiology of stem cell-derived neurons. For the electrophysiological recordings, cells were selected based on their neuronal morphology, and their identity determined post hoc by streptavidin-Alexa Fluor 488 labeling and immunostaining for $\alpha \mathrm{MSH}$ and NPY expression of the biotin ${ }^{+}$neurons. Current-clamp recordings were performed using a Multiclamp 700B amplifier. Data were digitized using a Digidata $1322 \mathrm{~A}$ analog-to-digital converter and were recorded at a $10-\mathrm{kHz}$ sample rate using pClamp 10 software (all equipment from Molecular Devices). Patch pipettes were fabricated using a P-97 pipette puller (Sutter Instruments). The external recording solution contained 145 $\mathrm{mM} \mathrm{NaCl}, 5 \mathrm{mM} \mathrm{KCl}, 10 \mathrm{mM}$ HEPES, $10 \mathrm{mM}$ glucose, $2 \mathrm{mM} \mathrm{CaCl}_{2}$, and $2 \mathrm{mM} \mathrm{MgCl}_{2}$. The $\mathrm{pH}$ was adjusted to 7.3 using $\mathrm{NaOH}$ and the osmolality adjusted to $325 \mathrm{mOsm}$ with sucrose. The pipette solution contained $130 \mathrm{mM} \mathrm{CH}_{3} \mathrm{KO}_{3} \mathrm{~S}, 10 \mathrm{mM} \mathrm{CH}_{3} \mathrm{NaO}_{3} \mathrm{~S}, 1 \mathrm{mM} \mathrm{CaCl}$, 10 mM EGTA, 10 mM HEPES, 5 mM MgATP, $0.5 \mathrm{mM} \mathrm{Na}_{2} \mathrm{GTP}$ (pH 7.3, $305 \mathrm{mOsm}$ ), and $0.1 \%$ biocytin hydrochloride. Experiments were performed at $21-23^{\circ} \mathrm{C}$. During recordings, current was applied to hold the cells at $-60 \mathrm{mV}$. Action potentials were evoked using incremental current steps 1 second in duration. The size of the current step increment was determined by the input resistance of the neuron, and each step was calculated (from Ohm's Law) to evoke a $5-\mathrm{mV}$ change in membrane potential. Analysis was carried out offline using Igor Pro 6 Software (WaveMetrics) with NeuroMatic (http://www.neuromatic. thinkrandom.com) and other custom-written procedures. Action potentials were counted automatically and were defined as events with a rise rate of greater than $20 \mathrm{mV} / \mathrm{ms}$, which reached a membrane potential greater than $0 \mathrm{mV}$.

RNA-seq. RNA was isolated from differentiated cells with QIAGEN RNAeasy Micro Kit. RNA quality was determined by 2100 Bioanalyzer at the Herbert Irving Comprehensive Cancer Center molecular biology facility at Columbia University. RNA-seq was performed by the Columbia Genome Center with a minimum of 30 million reads per sample. Hierarchical clustering was performed in Cluster (31) and visualized in Treeview (32). For each experiment and every gene, the gene expression values in FPKM were median centered and normalized to have unit variance before clustering. One matrix CIM (http:// discover.nci.nih.gov/cimminer/home.do) was used to generate the cluster heat map. All original microarray data were deposited in the NCBI's Gene Expression Omnibus (GEO GSE62936).

Neuropeptide assays. All the neurons were differentiated in a 12-well plate, starting with 300,000 cells that had been subjected to 12 days of the differentiation protocol per well. For neuron lysate samples, medium was aspirated and the differentiated neurons rinsed once with PBS, after which $250 \mu \mathrm{l} 0.1 \mathrm{~N} \mathrm{HCl}$ was added to each well. Cells were harvested with a cell scraper to detach as many cells as possible, and the cell lysates were transferred into $1.7 \mathrm{ml}$ Eppendorf tubes. These samples were sonicated for 5 minutes. After centrifugation at 10,000 $g$ for 10 minutes at $4^{\circ} \mathrm{C}$, the supernatants were collected for POMC, ACTH, $\alpha \mathrm{MSH}, \mathrm{BEP}$, and AGRP measurement. For medium samples, $1 \mathrm{ml}$ neuron culture medium was collected and centrifuged at $8,000 \mathrm{~g}$ for 5 minutes. Nine volumes of the supernatant $(720 \mu \mathrm{l})$ were mixed with 1 volume $1 \mathrm{~N} \mathrm{HCl}$ $(80 \mu \mathrm{l})$ to prepare a final $0.1 \mathrm{~N} \mathrm{HCl}$ sample mixture. For secretion studies, neurons were pretreated with neurobasal A (NBA) medium plus B27 (without insulin) overnight, then switched into NBA medium supplemented with either PBS or $60 \mathrm{mM} \mathrm{KCl}$ (final concentration). Twenty minutes later, medium was collected for ELISAs. Neurons from each well were lysed with RIPA buffer for protein assay. Secreted neuropeptides are expressed per $\mu$ g protein. AKT VIII inhibitor (Akti, $2 \mu \mathrm{M}$ ) was added to the N2 medium plus B27 and BDNF for 48 hours. Media and neuron lysates were collected for neuropeptide assay. Total POMC and BEP production were calculated by adding amounts of a neuropeptide in neuronal lysates to total released into the incubation medium. Neuropeptide assays were performed as described earlier $(35,37)$.

qPCR analysis. Cells were lysed with TRIzol RNA Reagent (Invitrogen) or RLT buffer. RNA was isolated by isopropanol precipitation or RNeasy Mini Kit (QIAGEN). cDNA were made with the GoScript Reverse Transcription System (Promega) with $1 \mu \mathrm{g}$ RNA for each sample. GoTaq qPCR Master Mix was used for qPCR (Promega). Human adult hypothalamus RNA was purchased from Clonetech. See Supplemental Table 1 for qPCR primers.

Western blot. Differentiated hESC/iPSC-derived neurons were pretreated with NBA medium (Life Technologies) plus B27 (without insulin, Life Technologies) for 16 hours and then exposed to $1 \mu \mathrm{g} / \mathrm{ml}$ insulin (Sigma-Aldrich) or $1 \mu \mathrm{g} / \mathrm{ml}$ leptin (Peprotech) with NBA medium/B27 (without insulin) for 30 minutes at $37^{\circ} \mathrm{C}, 5 \% \mathrm{CO}_{2}$. For Western blotting, cells and tissue were prepared as described (59). ACTIN was used as a loading control. See Supplemental Table 2 for antibody information.

Immunohistochemistry/immunocytochemistry. Cultured neurons were fixed with $4 \%$ PFA at room temperature (RT) for 15 minutes, then washed $3 \times$ with PBS for 5 minutes per wash. Cells were blocked with $10 \%$ donkey serum (Jackson ImmunoResearch) in PBST (PBS + 0.1\% Triton $\mathrm{X}-100)$ for 30 minutes, then switched into primary antibody (diluted with blocking buffer) solution and incubated at RT for 2 hours. Cells were washed $3 \times$ with PBST (5 minutes each wash), then exposed to secondary antibodies (Life Technology). Alexa Fluor secondary antibodies, goat or donkey anti-mouse, anti-rat, anti-rabbit, anti-goat, or anti-chicken $488,555,568$, and 647 were used at 1:400 dilution in PBST and incubated at RT for 1 hour. Following $3 \times$ wash with PBST, cells were incubated with Hoechst $(1 \mu \mathrm{g} / \mathrm{ml}$, diluted in PBST) for 
5 minutes and switched to PBS for fluorescent imaging. Details regarding these steps are presented in Supplemental Table 2. Teratoma sections $(8 \mu \mathrm{m})$ were $\mathrm{H} \& \mathrm{E}$ stained. Images were acquired with an Olympus IX71 epifluorescent microscope with an Olympus DP30BW black and white digital camera for fluorescence and a DP72 digital color camera for $\mathrm{H} \& \mathrm{E}$ staining. Some images were acquired using a Zeiss LSM710 confocal microscope or a Zeiss LSM5 Pascal microscope.

Flow cytometry. Flow cytometry was used to determine the differentiation efficiency from $\mathrm{hESC}$ to Nkx2.1-GFP-positive neuron progenitors. Day-12 differentiated cells were dissociated with Trypsin LE for 4 minutes at RT and all cells harvested into N2 medium. Cells were spun at $250 \mathrm{~g}$ for 4 minutes and the pellet rewashed with N2 medium. Cell pellets were resuspended in N2 medium plus B27 and $10 \mu \mathrm{M}$ ROCK inhibitor. Cells were filtered through a 5-ml polystyrene roundbottom tube with cell-strainer cap (Falcon) and FACS analyzed for $\mathrm{GFP}^{+}$cells with a BD Biosciences ARIA-IIu Cell Sorter. Cells without SHH and PM treatment were used as negative control. Flow cytometry data were analyzed using BD FACSDiva software.

Statistics. Unless otherwise indicated, all graphical data are presented as mean \pm SEM; significance was calculated using 2-tailed Student's $t$ tests. $P(\alpha)$ values of less than 0.05 were considered as significant.

Study approval. All human subjects provided written informed consent prior to their participation in this study. Human subject research was reviewed and approved by the Columbia Stem Cell Committee and the Columbia IRB. All animal studies were approved by the Columbia IACUC.

Note added in proof. In support of our findings, another group has also described the generation of hypothalamic neurons using similar strategies (60).

\section{Acknowledgments}

We thank Lisa Cole Burnett, Aiqun Li, Haiqing Hua, Kylie Foo, Andrew Sproul, and Samson Jacob for discussions related to this work; Andrew G. Elefanty (Monash University, Melbourne, Australia) for providing the Nkx2.1-GFP/W-hESC line; Florian Merkle and Kevin Eggan (Harvard University) for helpful discussions and information regarding antibodies; and L. Yang of the Diabetes and Endocrinology Research Center Pathology Core at Columbia University for cryosectioning. This research was supported by RO1 DK52431-20, the New York Stem Cell Foundation, a NYSTEM IIRP award (\#C026184), the Foundation for Prader-Willi Research, the Rudin Foundation, the Russell Berrie Foundation Program in Cellular Therapy, the Diabetes (P30 DK63608-12) and Obesity Research (P30 DK26687-33) Centers of Columbia University, an Institute of Human Nutrition predoctoral training grant (5 T32 DK00764725, RO1 DK 093920, New York State (N13G-275), New York State; N13G-275), and a Foundation Fighting Blindness New York Regional Research Center Grant (C-NY05-0705-0312). D. Egli is a NYSCF-Robertson investigator.

Address correspondence to: Dieter Egli or Rudolph Leibel, 1150 St. Nicholas Avenue, Room 620A, New York, New York 10032, USA, Phone: 212.851.4890, E-mail: de2220@cumc.columbia.edu (D. Egli). Phone: 212.851.5315; E-mail: rl232@cumc.columbia.edu (R. Leibel).

Matthew Freeby's present address is: Division of Endocrinology, Diabetes and Hypertension, UCLA Department of Medicine, Los Angeles, California, USA.
1. Pearson CA, Placzek M. Development of the medial hypothalamus: forming a functional hypothalamic-neurohypophyseal interface. Curr Top Dev Biol. 2013;106:49-88.

2. Alvarez-Bolado G, Paul FA, Blaess S. Sonic hedgehog lineage in the mouse hypothalamus: from progenitor domains to hypothalamic regions. Neural Dev. 2012;7:4

3. Barsh GS, Schwartz MW. Genetic approaches to studying energy balance: perception and integration. Nat Rev Genet. 2002;3(8):589-600.

4. Montague CT, et al. Congenital leptin deficiency is associated with severe early-onset obesity in humans. Nature. 1997;387(6636):903-908.

5. Krude H, Biebermann H, Luck W, Horn R, Brabant G, Gruters A. Severe early-onset obesity, adrenal insufficiency and red hair pigmentation caused by POMC mutations in humans. Nat Genet. 1998;19(2):155-157.

6. Farooqi IS, et al. Clinical and molecular genetic spectrum of congenital deficiency of the leptin receptor. NEngl J Med. 2007;356(3):237-247.

7. Farooqi IS, Keogh JM, Yeo GS, Lank EJ, Cheetham T, O'Rahilly S. Clinical spectrum of obesity and mutations in the melanocortin 4 receptor gene. N Engl JMed. 2003;348(12):1085-1095.

8. Yaswen L, Diehl N, Brennan MB, Hochgeschwender U. Obesity in the mouse model of pro-opiomelanocortin deficiency responds to peripheral melanocortin. Nat Med. 1999;5(9):1066-1070.
9. Kondo T, et al. Modeling Alzheimer's disease with iPSCs reveals stress phenotypes associated with intracellular $\mathrm{A} \beta$ and differential drug responsiveness. Cell Stem Cell. 2013;12(4):487-496.

10. Ryan SD, et al. Isogenic human iPSC Parkinson's model shows nitrosative stress-induced dysfunction in MEF2-PGC1 $\alpha$ transcription. Cell. 2013;155(6):1351-1364.

11. Kiskinis E, et al. Pathways disrupted in human ALS motor neurons identified through genetic correction of mutant SOD1. Cell Stem Cell. 2014;14(6):781-795.

12. Sternson SM. Hypothalamic survival circuits: blueprints for purposive behaviors. Neuron. 2013;77(5):810-824.

13. Maroof AM, et al. Directed differentiation and functional maturation of cortical interneurons from human embryonic stem cells. Cell Stem Cell. 2013;12(5):559-572.

14. Lu F, et al. Rax is a selector gene for mediobasal hypothalamic cell types. JNeurosci. 2013;33(1):259-272.

15. Hallonet M, et al. Vax1 is a novel homeoboxcontaining gene expressed in the developing anterior ventral forebrain. Development. 1998;125(14):2599-2610.

16. McNay DE, Pelling M, Claxton S, Guillemot F, Ang SL. Mash1 is required for generic and subtype differentiation of hypothalamic neuroendocrine cells. Mol Endocrinol. 2006;20(7):1623-1632.

17. Jing E, Nillni EA, Sanchez VC, Stuart RC, Good
DJ. Deletion of the Nhlh2 transcription factor decreases the levels of the anorexigenic peptides alpha melanocyte-stimulating hormone and thyrotropin-releasing hormone and implicates prohormone convertases I and II in obesity. Endocrinology. 2004;145(4):1503-1513.

18. Wang W, Lufkin T. The murine Otp homeobox gene plays an essential role in the specification of neuronal cell lineages in the developing hypothalamus. Dev Biol. 2000;227(2):432-449.

19. Michaud JL, DeRossi C, May NR, Holdener BC, Fan CM. ARNT2 acts as the dimerization partner of SIM1 for the development of the hypothalamus. Mech Dev. 2000;90(2):253-261.

20. Nakai S, et al. The POU domain transcription factor Brn-2 is required for the determination of specific neuronal lineages in the hypothalamus of the mouse. Genes Dev. 1995;9(24):3109-3121.

21. Majdic G, et al. Knockout mice lacking steroidogenic factor 1 are a novel genetic model of hypothalamic obesity. Endocrinology. 2002;143(2):607-614.

22. Szabo NE, Zhao T, Cankaya M, Theil T, Zhou X, Alvarez-Bolado G. Role of neuroepithelial Sonic hedgehog in hypothalamic patterning. J Neurosci. 2009;29(21):6989-7002.

23. Liu Y, et al. Medial ganglionic eminence-like cells derived from human embryonic stem cells correct learning and memory deficits. Nat Biotechnol. 2013;31(5):440-447.

24. Wataya T, et al. Minimization of exogenous 
signals in ES cell culture induces rostral hypothalamic differentiation. Proc Natl Acad Sci U S A. 2008;105(33):11796-11801.

25. Chambers SM, Fasano CA, Papapetrou EP, Tomishima M, Sadelain M, Studer L. Highly efficient neural conversion of human ES and iPS cells by dual inhibition of SMAD signaling. Nat Biotechnol. 2009;27(3):275-280.

26. Aujla PK, Naratadam GT, Xu L, Raetzman LT. Notch/Rbpjк signaling regulates progenitor maintenance and differentiation of hypothalamic arcuate neurons. Development. 2013;140(17):3511-3521.

27. Ratie L, et al. Novel genes upregulated when NOTCH signalling is disrupted during hypothalamic development. Neural Dev. 2013;8:25.

28. Nelson BR, Hartman BH, Georgi SA, Lan MS, Reh TA. Transient inactivation of Notch signaling synchronizes differentiation of neural progenitor cells. Dev Biol. 2007;304(2):479-498.

29. Padilla SL, Carmody JS, Zeltser LM. Pomcexpressing progenitors give rise to antagonistic neuronal populations in hypothalamic feeding circuits. Nat Med. 2010;16(4):403-405.

30. Hamill OP, Marty A, Neher E, Sakmann B, Sigworth FJ. Improved patch-clamp techniques for high-resolution current recording from cells and cell-free membrane patches. Pflugers Arch. 1981;391(2):85-100.

31. de Hoon MJ, Imoto S, Nolan J, Miyano S. Open source clustering software. Bioinformatics. 2004;20(9):1453-1454.

32. Saldanha AJ. Java Treeview - extensible visualization of microarray data. Bioinformatics. 2004;20(17):3246-3248.

33. Zhu X, Gleiberman AS, Rosenfeld MG. Molecular physiology of pituitary development: signaling and transcriptional networks. Physiol Rev. 2007;87(3):933-963.

34. Savage JJ, Yaden BC, Kiratipranon P, Rhodes SJ. Transcriptional control during mammalian anterior pituitary development. Gene. 2003;319:1-19.

35. Sei C, Toneff T, Aaron W, Hook VY. Regulation of cellular $\alpha$-MSH and $\beta$-endorphin during stimulated secretion from intermediate pituitary cells: involvement of aspartyl and cysteine proteases in the control of cellular levels of $\alpha$-MSH and $\beta$-endorphin. Peptides. 2002;23(8):1409-1418.

36. Wardlaw SL. Hypothalamic proopiomelanocortin processing and the regulation of energy balance. Eur J Pharmacol. 2011;660(1):213-219.

37. Page-Wilson G, et al. Cerebrospinal fluid levels of leptin, proopiomelanocortin, and agoutirelated protein in human pregnancy: evidence for leptin resistance. JClin Endocrinol Metab. 2013;98(1):264-271.

38. Rius RA, Chikuma T, Loh YP. Prenatal processing of pro-opiomelanocortin in the brain and pituitary of mouse embryos. Brain Res Dev Brain Res. 1991;60(2):179-185

39. Grossman HC, Hadjimarkou MM, Silva RM, Giraudo SQ, Bodnar RJ. Interrelationships between mu opioid and melanocortin receptors in mediating food intake in rats. Brain Res. 2003;991(1):240-244.

40. Breen TL, Conwell IM, Wardlaw SL. Effects of fasting, leptin, and insulin on AGRP and POMC peptide release in the hypothalamus. Brain Res. 2005;1032(1):141-148.

41. Plum L, Belgardt BF, Bruning JC. Central insulin action in energy and glucose homeostasis. JClin Invest. 2006;116(7):1761-1766.

42. Baskin DG, et al. Insulin and leptin: dual adiposity signals to the brain for the regulation of food intake and body weight. Brain Res. 1999;848(1):114-123.

43. Bates SH, et al. STAT3 signalling is required for leptin regulation of energy balance but not reproduction. Nature. 2003;421(6925):856-859.

44. Odawara A, Saitoh Y, Alhebshi AH, Gotoh M, Suzuki I. Long-term electrophysiological activity and pharmacological response of a human induced pluripotent stem cell-derived neuron and astrocyte co-culture. Biochem Biophys Res Commun. 2014;443(4):1176-1181.

45. Sherafat-Kazemzadeh R, et al. Hyperphagia among patients with Bardet-Biedl syndrome. Pediatr Obes. 2013;8(5):e64-e67.

46. Ovesjo ML, Gamstedt M, Collin M, Meister B. GABAergic nature of hypothalamic leptin target neurones in the ventromedial arcuate nucleus. JNeuroendocrinol. 2001;13(6):505-516.

47. Gill R, et al. Whole-exome sequencing identifies novel LEPR mutations in individuals with severe early onset obesity. Obesity (Silver Spring). 2014;22(2):576-584.

48. Wheeler E, et al. Genome-wide SNP and CNV analysis identifies common and low-frequency variants associated with severe early-onset obesity. Nat Genet. 2013;45(5):513-517.

49. Paz-Filho G, et al. Whole exome sequencing of extreme morbid obesity patients: translational implications for obesity and related disorders. Genes (Basel). 2014;5(3):709-725.

50. Clee SM, Attie AD. The genetic landscape of type 2 diabetes in mice. Endocr Rev. 2007;28(1):48-83.

51. Coleman DL, Hummel KP. The influence of genetic background on the expression of the obese (Ob) gene in the mouse. Diabetologia. 1973;9(4):287-293.

52. Leibel RL, Chung WK, Chua SC, Chua SC Jr. The molecular genetics of rodent single gene obesities. J Biol Chem. 1997;272(51):31937-31940.

53. Comuzzie AG, Allison DB. The search for human obesity genes. Science. 1998;280(5368):1374-1377.

54. Hockemeyer D, et al. Efficient targeting of expressed and silent genes in human ESCs and iPSCs using zinc-finger nucleases. Nat Biotechnol. 2009;27(9):851-857.

55. Ran FA, Hsu PD, Wright J, Agarwala V, Scott DA, Zhang F. Genome engineering using the CRISPRCas9 system. Nat Protoc. 2013;8(11):2281-2308.

56. Hua H, et al. iPSC-derived beta cells model diabetes due to glucokinase deficiency. J Clin Invest. 2013;123(7):3146-3153.

57. Dimos JT, et al. Induced pluripotent stem cells generated from patients with ALS can be differentiated into motor neurons. Science. 2008;321(5893):1218-1221.

58. Albuquerque C, Joseph DJ, Choudhury P, MacDermott AB. Dissection, plating, and maintenance of dorsal root ganglion neurons for monoculture and for coculture with dorsal horn neurons. Cold Spring Harb Protoc. 2009;2009(8):pdb prot5275.

59. Qiang L, et al. Brown remodeling of white adipose tissue by SirT1-dependent deacetylation of Ppargamma. Cell. 2012;150(3):620-632.

60. Merkle F, et al. Generation of neuropeptidergic hypothalamic neurons from human pluripotent stem cells. Development. In press. 